\title{
Coronal phenomena at the release of solar energetic electron events ${ }^{\star}$
}

\author{
K.-L. Klein ${ }^{1}$, S. Krucker ${ }^{2}$, G. Trottet ${ }^{1}$, and S. Hoang ${ }^{1}$ \\ 1 Observatoire de Paris, LESIA-CNRS UMR 8109, 92195 Meudon, France \\ e-mail: ludwig.klein@obspm.fr \\ 2 Space Sciences Laboratory, University of California, Berkeley, CA 94720-7450, USA \\ e-mail: krucker@ssl.berkeley.edu
}

Received 7 May 2004 / Accepted 9 October 2004

\begin{abstract}
We investigate dynamical processes in the solar corona at the release of electrons $(\sim 30-500 \mathrm{keV})$ detected by the Wind/3DP experiment, with the aim to clarify the relationship between coronal acceleration and the escape of electrons to interplanetary space. Energetic electrons and plasma in the corona are traced using radio, EUV and X-ray observations. 40 events were identified where the release time of the electrons could be determined within an uncertainty of a few minutes and occurred during the observing hours of the Nançay Radioheliograph. All were accompanied by decametric-to-kilometric type III bursts (Wind/WAVES), and 30 by metric radio emission in the western hemisphere. The main findings from these 30 events are: (i) Electrons detected at Wind are released at the time of distinct episodes of electron acceleration in the corona signalled by radio emission. The release may occur at the start of the radio event or up to an hour later. (ii) The most conspicuous examples of delayed electron release occur in events associated with complex, long lasting ( $>10 \mathrm{~min}$, up to seveal hours) radio emission. Radio observations suggest that in these cases the earlier accelerated electrons remain confined in the corona or are injected into flux tubes which are not connected to the spacecraft. (iii) Type II bursts revealing shock waves in the corona accompany about a third of the events. But the shock waves occur in general together with type IV radio signatures due to long lasting acceleration not related to the shock. With a few exceptions these type IV emissions have a clearer timing relationship with the electron release to space than the type II bursts. We conclude that the combination of time-extended acceleration at heights $\lesssim 0.5 R_{\odot}$ above the photosphere with the injection of electrons into a variety of closed and open magnetic field structures explains the broad variety of timing shown by the radio observations and the in situ measurements.
\end{abstract}

Key words. Sun: coronal mass ejections (CMEs) - Sun: flares - Sun: particle emission - Sun: radio radiation

\section{Introduction}

Solar energetic particle (SEP) events accompany flares, i.e. explosive energy conversion within active regions, and coronal mass ejections (CMEs), revealing the reconfiguration of the coronal magnetic field on much larger scales. Both types of activity provide possibilities for particle acceleration, related to magnetic reconnection and to large-scale coronal shock waves. The timing of the particle release at the Sun can in principle be used to identify the most relevant coronal processes at the time of acceleration. Contemporary spaceborne and groundbased instrumentation provides timing with an accuracy of a few minutes. Using the Wind and ACE spacecraft, respectively, Krucker et al. (1999) and Haggerty \& Roelof (2002) concluded that the release of electrons into interplanetary space often starts several minutes after the first radiative signatures of electrons in the corona. The late release was ascribed to the action of a different acceleration process for the escaping electrons,

* Appendix A is only available in electronic form at http://www.edpsciences.org related to shocks associated with flares or CMEs (Krucker et al. 1999; Simnett et al. 2002). An alternative to late acceleration is the delay by transport (Kallenrode \& Wibberenz 1990). Cane (2003) compared the arrival times of electrons and of the type III emission at the spacecraft for the event sample of Haggerty \& Roelof (2002) and concluded that the delays were indeed produced by interplanetary propagation.

A different accelerator of the escaping electrons, such as a shock wave, or transport which delays the electrons' arrival at the spacecraft, are required to explain such delays especially under two assumptions: that flare-related electron acceleration in the corona is a very short-lasting process, e.g. of minutesduration, typical of the impulsive phases of solar flares, and that the accelerated particles are guided into interplanetary space along a unique flux tube to which a spacecraft is either connected or not. But it is known that these hypotheses are not, or at least not always, valid. Coronal electron acceleration associated with flares may last tens of minutes or even several hours, as shown by gamma-ray, hard X-ray and radio emissions 
(see Hudson 1978; Trottet 1986; Cliver et al. 1986; Kocharov et al. 1994; Akimov et al. 1996; Klein \& Trottet 2001). And most metre-wave type III bursts, which trace electron beams travelling outward from active regions, reveal beam injection into various bundles of diverging field lines with overall opening angles of several tens of degrees (Pick \& Ji 1986). It is also well established that even apparently simple decametrichectometric (henceforth DH) type III bursts are associated with groups of type III bursts at higher frequencies (Poquérusse et al. 1996). It is then possible that the first electrons accelerated in the corona are not released onto field lines connected with a given spacecraft, while later releases occur on well-connected flux tubes. In this case delays would be due to incomplete sampling of the accelerated electrons by the spacecraft, not to the acceleration or propagation of the electrons. In an attempt to test if this spatial and temporal complexity of electron acceleration in flaring active regions is relevant to the timing of electrons detected in space, we present here a systematic study of the energetic electrons in the corona during the release of electrons above $30 \mathrm{keV}$ detected at Wind. A preliminary report has been given by Klein et al. (2003a). A similar study using electron detection with the ACE spacecraft was carried out by Pick et al. (2003) and Maia \& Pick (2004).

In Sect. 2 the instruments used and the event selection are first presented. Then an overview on the results for 30 electron events accompanied by metre wave emission is given separately for electron events related to radio emission of short and long duration. For more detailed examples the reader is referred to the Appendix. The results are discussed in Sect. 3 in terms of time-extended acceleration in the corona and propagation of the electrons into interplanetary space in diverging magnetic flux tubes.

\section{Observations and analysis}

\subsection{Instruments}

The Three-dimensional Plasma and Energetic Particles Instrument on the Wind spacecraft (henceforth Wind/3DP; Lin et al. 1995) measures three-dimensional distribution functions of electrons in the range $\sim(30-500) \mathrm{keV}$, using a system of silicon semiconductor detectors. Electrostatic detectors provide additional measurements of electrons from solar wind energies to $\sim 30 \mathrm{keV}$. The Nançay Radioheliograph (Kerdraon \& Delouis 1997, NRH in the following) maps radio emission routinely at five frequencies between 164 and $432 \mathrm{MHz}$, at a rate of 8 images per second and frequency, with a spatial resolution of $\sim 3^{\prime}$ at $164 \mathrm{MHz}$. The spectral identification at decimetric-to-metric wavelengths is based on reports of the IZMIRAN, Potsdam and Zürich observatories published by NOAA in Solar Geophys. Data or available via the web-pages ${ }^{1}$. Decametric spectra $(20-70 \mathrm{MHz})$ were provided by the Nançay Decametric Array (henceforth NDA; Lecacheux 2000), and decametric-to-kilometric spectra by the WAVES experiment aboard the Wind spacecraft

\footnotetext{
${ }^{1}$ http://helios.izmiran.rssi.ru/lars/LARS.html, http://www.aip.de/groups/osra/, http://www . astro.phys.ethz.ch/rapp/
}

(Bougeret et al. 1995, http://lep694.gsfc.nasa.gov/ waves/waves.html). The spacecraft spin modulation of the received signal is used to measure the direction of the source centroid and the source width at frequencies below $1 \mathrm{MHz}$ (Manning \& Fainberg 1980); the effects of the propagation, of the source dimension and interplanetary density structures on these parameters were discussed by Reiner (2001). The coronal plasma structures to which the radio emissions are related are traced by EUV imaging from the Extreme Ultraviolet Imaging Telescope (EIT; Delaboudinière et al. 1995, http://sohowww.nascom.nasa.gov/) on the Solar and Heliospheric Observatory (SoHO) or by the soft X-ray imaging from Yohkoh (Tsuneta et al. 1991, http://www . lmsal . com/SXT/homepage.html). The time history of the whole Sun soft X-ray flux observed by the Geostationary Operational Environmental Satellite (GOES, data provided by the Solar Data Analysis Center at NASA Goddard Space Flight Center, http:// umbra.nascom.nasa.gov/) was used as a tracer of the temporal evolution of energy release in the low corona.

\subsection{Selection of electron events and determination of the release time}

Electron events were selected in the Wind/3DP data base

- which showed a clear anisotropy at their onset revealing antisunward streaming;

- whose solar release time could be inferred from the dispersion of the arrival times of electrons in different energy channels from $\sim 30$ to $500 \mathrm{keV}$ at the spacecraft to within an uncertainty less than $10 \mathrm{~min}$;

- whose solar release occurred during the observing time of the NRH.

For the onset time determination we did not consider energies below $30 \mathrm{keV}$, because the electrostatic detectors have relatively high background. Like Haggerty \& Roelof (2002), we refer to the electrons above $30 \mathrm{keV}$ as "near-relativistic" in the following. Their arrival times at the spacecraft were determined for each energy channel in the sunward direction. The release time and the length of the interplanetary trajectory were inferred from a linear fit to the arrival time as a function of inverse speed, under the assumption that electrons at all energies are released simultaneously (see Krucker et al. 1999, for details). When only three energy channels showed a clear event onset, or when the analysis yielded a very large uncertainty or an unplausible path length, the release times were determined by assuming that the path length is equal to the Parker spiral length. As a result, we obtain the most probable release time within a time interval given by the estimated uncertainty. This time interval, which roughly corresponds to an uncertainty of $3 \sigma$, is called the release window in the following. It identifies the solar release time of all electrons when the release is of very short duration as compared to the interplanetary travel time, and the start of the solar release when it is time-extended.

The release times inferred by these methods are estimated to be accurate to within a few minutes on average. The uncertainty is greater in weak events or events with high background. 
Systematic errors are not considered. For example, a fundamental hypothesis is that electrons of all energies start at the Sun within a time interval that is negligibly short compared to their shortest interplanetary travel time, and are released from a volume that is small compared to the distance to the spacecraft. Deviations from this hypothesis become noticeable when an unrealistic interplanetary path length results from the fit of arrival times. Since the derived path lengths are close to the nominal Parker spiral length, we expect that the error of the release time induced by energy dependence is in general negligible. An important source of systematic errors is the detector background noise. Since the release window is derived from the times when the electron count rates start to exceed background in the different energy channels, the steepness of the rise of the count rates affects this estimation. A shallow rise will induce a larger systematic error than a steep slope, and might create an artificial delay of the event onset by up to a few minutes. Interplanetary scattering will also reduce the number of promptly arriving electrons at the spacecraft, and might further contribute to hide the event onset in the background noise (Kallenrode \& Wibberenz 1990). Finally, spatial effects which are not strong enough to eliminate the event from the beginning may confuse the onset time. These systematic delays are not expected to exceed a few minutes.

For all events the NRH images were examined for activity in the vicinity of the electron release window. Reevaluation of the events discussed in Klein et al. (2003a) led to the elimination of 17 cases where the onset time determination was uncertain due to a high pre-event background, or which were detected in too few energy channels or did not display a beamed distribution at their onset. A sample of 40 beamed events whose release window could be reliably determined and fell into the observing time of the NRH was identified between December 1996 and May 2001.

\subsection{Observations}

In 10/40 events the NRH maps showed either no nonthermal emission at all at any frequency, or emission from the eastern hemisphere which had no obvious relation with the electron event, or long lasting noise storm emission which existed well before the electron release. More details are given in the Appendix (Sect. A.3). Hence three quarters of the electron events exhibited some metric radio emission (i) at longitudes where electrons may gain access to the nominal Earthconnected interplanetary magnetic field line; and (ii) at times within or, following the above discussion of systematic errors, close to, the electron release window.

The different kinds of metre wave emission reveal different types of exciters and magnetic structures in the corona. A detailed description of metre wave radio bursts was given by McLean \& Labrum (1985). Most emissions are thought to occur near the local plasma frequency or its harmonic, which means for NRH observations a range of ambient electron densities from $2.5 \times 10^{9} \mathrm{~cm}^{-3}$ (plasma frequency $450 \mathrm{MHz}$, wavelength $67 \mathrm{~cm})$ to $7.0 \times 10^{7} \mathrm{~cm}^{-3}(75 \mathrm{MHz}, 4 \mathrm{~m})$. The electromagnetic waves are produced through the coupling of
Langmuir waves or upper hybrid waves excited by unstable distributions of energetic electrons. Type III emission arises from Langmuir waves generated by electron beams travelling upward through the corona in open magnetic flux tubes at a typical speed $\sim 0.25 c$ (energy $\sim 15 \mathrm{keV}$ ). Type II bursts are excited by electrons accelerated at shock waves. As the shock proceeds to regions of decreasing ambient density, the emission drifts towards lower frequencies at a slower rate than type III bursts. Type IV bursts are broadband emissions with duration up to several hours. Most type IV bursts are also attributed to plasma emission by electrons in closed large-scale magnetic structures, where a range of plasma frequencies is excited simultaneously (see Pick 1986, for a detailed presentation). Gyrosynchrotron emission from mildly relativistic electrons can explain type IV bursts with structureless spectra and moderate brightness, but not the bright emissions which stand out in dynamic spectrograms. Noise storms have a similar spectral structure, but occur without direct relationship to flares or CMEs. Short, narrowband bursts within noise storms are referred to as "type I" bursts.

The 30 radio events associated with near relativistic electron releases were divided into two categories, depending on the duration of the metric radio emission observed by the NRH: (i) events where the release window comprised or was close to a short group of bursts, most often including type III bursts; (ii) events where the release window occurred during metric radio emission of long duration (typically $>10 \mathrm{~min}$, up to several hours). The metric emission of this category had most often several components, always including some kind of type IV continuum. The division in terms of duration is related to the duration of electron acceleration. The electrons of up to tens of $\mathrm{keV}$ which probably emit the bright type IV bursts and the noise storms must be accelerated over a duration comparable with that of the radio emission. For the collisional lifetime of electrons with energy $E$ of some tens of $\mathrm{keV}$ in a plasma with electron density $n_{0}$ is $\tau[\mathrm{s}]=\left(\frac{1.4 \times 10^{8} \mathrm{~cm}^{-3}}{n_{0}}\right)\left(\frac{E}{1 \mathrm{keV}}\right)^{1.5}$ (after Bai \& Ramaty 1979). The relevant ambient electron density for emission at $164 \mathrm{MHz}$ is of order $10^{8} \mathrm{~cm}^{-3}$. Hence in the long radio events (duration $\geq 10$ min to several hours) only relativistic electrons might survive in coronal traps, not the electrons at lower energies which generate the coherent emissions.

\subsection{Near-relativistic electron releases associated with short metric radio emission}

\subsubsection{May 1: A short duration radio event with prompt electron release}

The electron and electromagnetic signatures of a typical event with short radio emission are illustrated in Fig. 1. The time profiles of the near-relativistic electron count rates are plotted in Fig. $1 \mathrm{~b}$ in the directions along $\left(0^{\circ}\right)$ and opposite $\left(180^{\circ}\right)$ to the local magnetic field direction, which was sunward in this case. Electrons streaming from the Sun are in the $180^{\circ}$ direction. The top panel shows the pitch angle distribution of (30-50) $\mathrm{keV}$ electrons. For each time bin, the flux values are normalised by the pitch angle averaged flux. Times with isotropic 


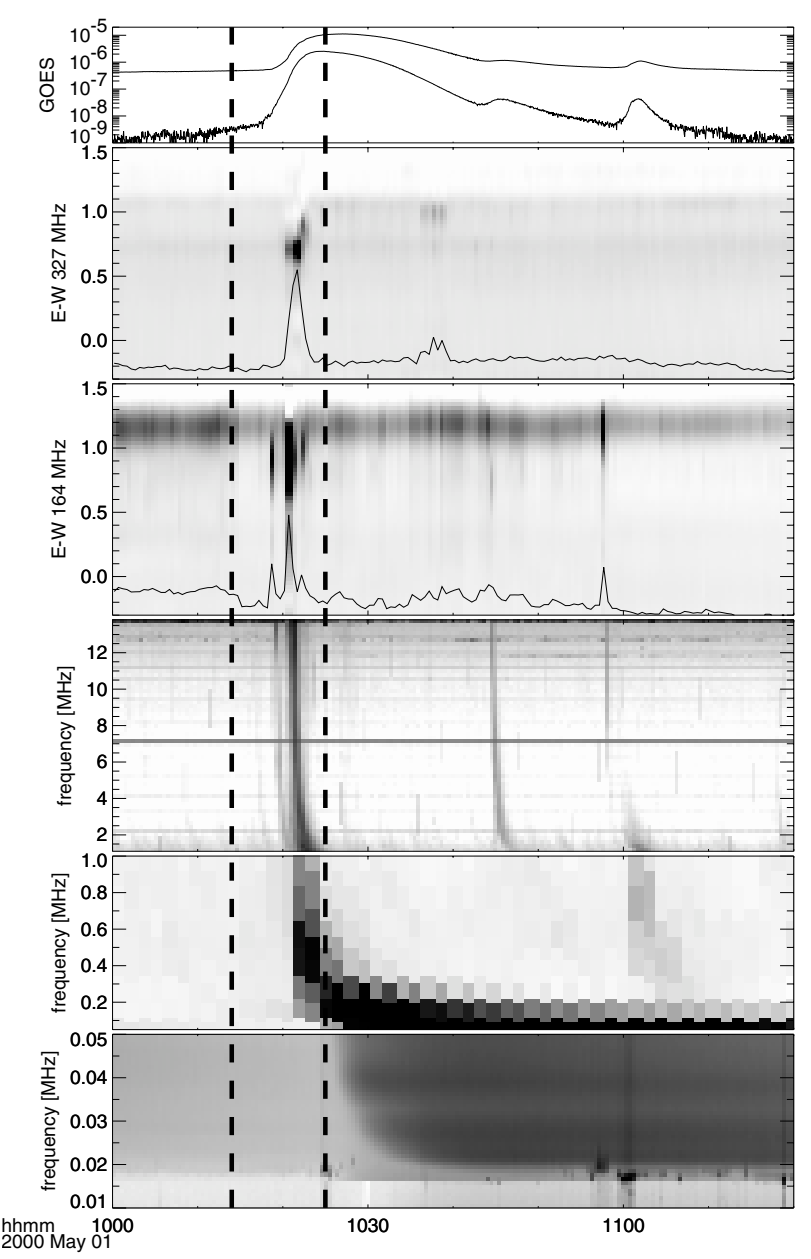

(a)

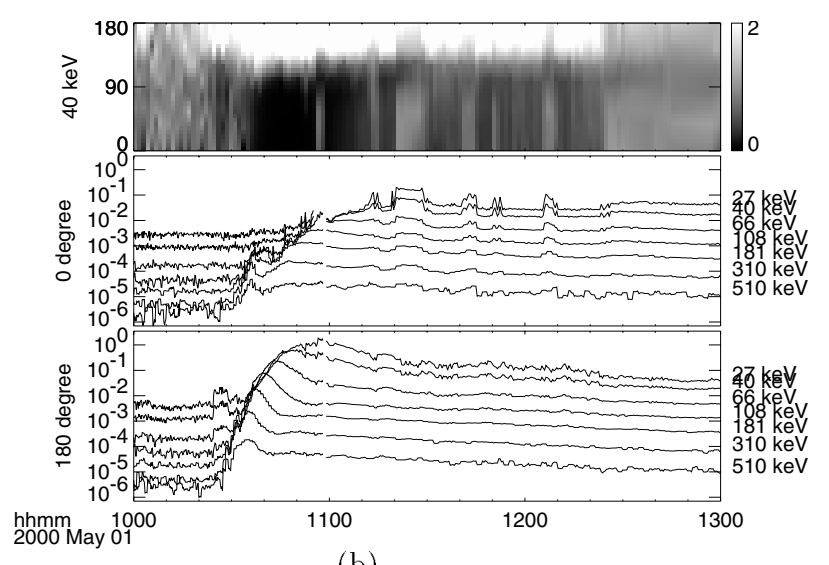

(b)

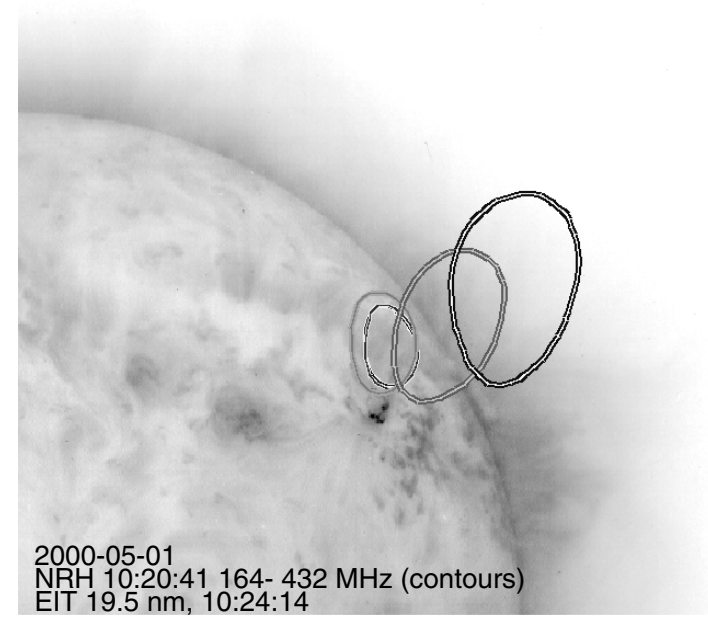

(c)

Fig. 1. 2000 May 1: a) time histories of (from top to bottom) the soft X-ray flux in the (0.1-0.8) nm (top) and (0.05-0.4) nm ranges (GOES), the radio brightness (NRH) integrated over the solar south-north direction at $327 \mathrm{MHz}$ (second from top) and $164 \mathrm{MHz}$ (third from top; ordinate graded in units of a solar radius, from east $(<0)$ over disk centre $(0)$ to west; reversed colour scale indicating bright emission by dark shading), the dynamic radio flux density spectrum at decametric to kilometric waves (Wind/WAVES). The vertical dashed lines delimit the release window of the electrons detected by Wind/3DP. b) Electrons at Wind/3DP; top: pitch angle distribution for 30-50 keV electrons. Times with isotropic distributions correspond to values around 1 (grey), beamed distributions show larger values in the beam direction (white), lower values in the opposite direction (black). c) Superposition of the metric radio sources, represented by the iso-intensity lines at half maximum at 432, 327, 237, $164 \mathrm{MHz}$, on top of a nearly simultaneous EIT image $(\lambda=19.5 \mathrm{~nm}$; reversed colour table). Solar north is at the top, west to the right.

distributions correspond to values around 1 (grey), beamed distributions show larger values (white) in the beam direction, lower values (black) in the opposite direction. The white shading in the pitch angle-time-plane hence shows that the electrons are beamed along the interplanetary magnetic field. The release window of these electrons is delimited by the two vertical dashed lines in Fig. 1a, and compared with electromagnetic signatures in the corona. The figure shows, from top to bottom, the GOES soft X-ray fluxes in the $(0.1-0.8) \mathrm{nm}$ and $(0.05-0.4) \mathrm{nm}$ channels (units $\mathrm{W} \mathrm{m}^{-2}$ ), grey-scale plots of one-dimensional brightness at metre wavelengths (327 and $164 \mathrm{MHz})$, and the dynamic spectrum from decametric to kilometric wavelengths. The 1D metre wave brightness is obtained by integrating each map along the solar south-north direction. This way one eastwest scan is obtained for each time bin, in this case using $10 \mathrm{~s}$ integration. The vertical axis of the $1 \mathrm{D}$ brightness plot gives the east-west coordinate in solar radii, the zero is the centre of the disk. From the relative timing and the spectral classification of the different electromagnetic emissions this was a typical impulsive flare.

To within the uncertainties of the method, the nearrelativistic electrons detected at $\mathrm{Wind} / 3 \mathrm{DP}$ were released at the time of a DH type III burst, metre wave emission, and the rise of the soft X-ray flux. The metre wave emission is identified as type III at frequencies below $270 \mathrm{MHz}$ (IZMIRAN, Solar Geophys. Data). Three strong bursts stand out during the release window in the $1 \mathrm{D}$ brightness plot at $164 \mathrm{MHz}$. The third one has a source slightly west of the first two. The dark band centred at West $1.2 R_{\odot}$ is a noise storm, which has no evident relationship with the electron event under study. At frequencies above $270 \mathrm{MHz}$ (e.g., $327 \mathrm{MHz}$ in Fig. 1a), the time profile at $1 \mathrm{~s}$ resolution shows no evidence for individual type III bursts, 
but resembles decimetric broadband emission and spikes which are often seen above the high frequency limit of type III bursts (e.g., Paesold et al. 2001, and references therein). Like in the type III emission at $164 \mathrm{MHz}$, the successive brightening at two positions is also seen in the $1 \mathrm{D}$ brightness at $327 \mathrm{MHz}$.

The type III emission at frequencies between $14 \mathrm{MHz}$ and $1 \mathrm{MHz}$ is the low-frequency continuation of the metric type III bursts. The bursts merged into a single one below $1 \mathrm{MHz}$. Its low-frequency border (bottom panel of Fig. 1a) approached the local plasma frequency at the spacecraft, characterised here by the dark (i.e. bright) line seen in the TNR spectrum near $15 \mathrm{kHz}$. The brightest, short-lived features near $15 \mathrm{kHz}$ are rapidly varying emissions that we identify as packets of Langmuir waves. Since type III bursts are generated indirectly via the production of Langmuir waves by electron beams, and since Langmuir waves cannot escape from the source, their detection at Wind is a hint that the spacecraft actually intercepted the electron beam producing the type III emission (Lin et al. 1986; Hoang et al. 1994). In the present case the spacecraft was connected to the Earth's bow shock and might have observed Langmuir waves from electron beams produced there. But since the number of Langmuir wave detections increased when the type III emission approached the plasma line, we believe that some are produced by the type III electron beams coming from the Sun. This confirms the magnetic connection of the spacecraft to the aceleration region of the type III emitting electron beams. They must have travelled along the same magnetic flux tube as the near-relativistic electrons detected at Wind/3DP, which in turn implies that both came from the same accelerator.

Figure 1c displays a snapshot map of the metric radio sources, represented by their iso-intensity contours at half maximum $(432,327,237,164 \mathrm{MHz}$, in the order of increasing darkness). They are overplotted on an EIT $19.5 \mathrm{~nm}$ image taken less than four minutes later. The radio sources overlay an active region where a flare showed up as a compact brightening in EUV (dark spots south of the high-frequency radio sources in Fig. 1c). The positions of the radio sources are consistent with plasma emission from electron beams that travel in magnetic flux tubes connected with the flaring active region. The two distinct and successively brightening metre wave sources indicate that electron beams were successively injected into different flux tubes rooted in closely neighbouring sites in the low atmosphere. The start of the type III emission between the two neighbouring NRH frequencies 327 and $237 \mathrm{MHz}$ suggests that the electron beams which escaped into interplanetary space were accelerated between the sources at these frequencies, i.e. within a fraction of a solar radius above the photosphere.

The flare was accompanied by a CME observed by LASCO with speed $1360 \mathrm{~km} \mathrm{~s}^{-1}$ in Yashiro's LASCO catalogue (Catholic University of America and NASA GSFC, http://cdaw.gsfc.nasa.gov/CME_list) and $933 \mathrm{~km} \mathrm{~s}^{-1}$ in Simnett et al. (2002). These values imply that the front was at a heliocentric distance of $(1.4-2.0) R_{\odot}$ at the time of the DH type III burst, well ahead of the metric type III radio sources and of the presumable acceleration site of the escaping electrons. If the CME did drive a shock, as suggested by its high speed, this shock was either not yet formed at the time of the electron release, or it was transparent to the escaping electrons.

\subsubsection{Overview of the sample associated with short metric radio emission}

Table 1 lists the electron events associated with short metre wave emission. The format is as follows:

1. The first column gives the date (first line), the release window (second line; $500 \mathrm{~s}$ added so that it can be directly compared with the arrival time of photons at $1 \mathrm{AU}$ ) and its delay (third line, within parentheses) with respect to the associated DH type III emission. The first number is the difference between the start of the release window and the end of the DH type III emission that we associated with the electron event (Col. 3), the second number is the difference between the end of the release window and the start of the type III group. This manner of defining delays leads to rather large uncertainties, but there is no a priori reason to consider the start of a group of type III bursts as the only relevant quantity for comparison with the electron release. In cases where several DH type III bursts or groups were detected, the fourth line gives the delay of the release window with respect to the closest burst or group.

2. The second column gives the energy range where the electron event could be identified by the Wind/3DP detector.

3. The "(0-14) MHz" column lists the starting frequency and approximate time (single bursts) or time interval (groups) of DH type III bursts in the high-frequency band of the WAVES instrument. Type II or type IV bursts indicated in the event table on the WAVES GSFC homepage are also listed in this column with the time and frequency where they started. The last line indicates if Langmuir waves were detected at the spacecraft: "possible L" marks ambiguous cases, "BS" marks events where the spacecraft was possibly connected with the Earth's bow shock, where Langmuir waves are also generated by locally produced electron beams.

4. In the "(20-70) MHz" column observations of the Nançay Decametric Array are listed when available. If no time is given, the type III burst is the extension towards higher frequencies of the burst detected by WAVES.

5. The fifth column gives the spectral type (from spectrograph reports or, in the case of type IV bursts and noise storms, from the NRH data), time interval and approximate location (heliocentric distance in solar radii and position angle counted counterclockwise from north, rounded to the nearest multiple of $5^{\circ}$ ) of the radio source at $164 \mathrm{MHz}$ during the electron release window and the DH type III emission.

6. The column "flare position" and "SXR" lists the location (heliographic latitude and longitude), importance and starting time of flares in $\mathrm{H} \alpha$ reported during the $\mathrm{DH}$ type III bursts or the release window in Solar Geophys. Data. Alternatively, the coordinates of brightenings in EIT images $(19.5 \mathrm{~nm})$ are given. Flares in the eastern hemisphere are quoted within parentheses. In the last line of each entry in this column, the importance, start and peak time of the 
Table 1. 3DP events related to short metre-wave emission.

\begin{tabular}{|c|c|c|c|c|c|c|}
\hline $\begin{array}{l}\text { Release } \\
\text { window }\end{array}$ & $\begin{array}{r}E \text { range } \\
{[\mathrm{keV}]} \\
\end{array}$ & $\begin{array}{r}(0-14) \mathrm{MHz} \\
(\text { WAVES) } \\
\end{array}$ & $\begin{array}{r}(20-70) \mathrm{MHz} \\
(\mathrm{NDA}) \\
\end{array}$ & $\begin{array}{r}\geq 164 \mathrm{MHz} \\
(\mathrm{NRH}) \\
\end{array}$ & $\begin{array}{l}\text { Flare position } \\
\text { SXR (GOES) }\end{array}$ & $\begin{array}{r}\mathrm{CME} \\
\mathrm{PA}, \Delta, v\end{array}$ \\
\hline 1996 Dec. 24 & $30-\geq 500$ & III $>14 \mathrm{MHz}$ & No data & III, II & EIT 13:14 W limb & 26569325 \\
\hline $13: 08-13: 18$ & & $13: 04-13: 24$ & & $13: 03-13: 16$ & C2.1 13:03 13:11 & \\
\hline$(-16+14)$ & & L waves & & $1.2280^{\circ}$ & & \\
\hline 1997 Nov. 23 & $\sim 1-200$ & III $>14 \mathrm{MHz}$ & No data & III & N19W45 SF 12:54 & None \\
\hline $12: 53-12: 59$ & & $12: 47$ & & $12: 47-12: 53$ & B6.1 12:51 12:59 & Reported \\
\hline$(+6+12)$ & & possible L & & $1.3295^{\circ}$ & & \\
\hline 1998 Jul. 11 & $\sim 1-300$ & III $>14 \mathrm{MHz}$ & III 20-70 & III & N16W67 SF 12:49 & No data \\
\hline $12: 52-12: 56$ & & $12: 47-12: 51$ & $12: 47-12: 49$ & $12: 47-12: 51$ & C4.3 12:43 12:50 & \\
\hline$(+1+9)$ & & L waves & & $1.3285^{\circ}$ & & \\
\hline 1998 Sep. 27 & $\sim 5-\geq 500$ & III $>14 \mathrm{MHz}$ & III 20-70 & III & N21W48 SF 08:09 & No data \\
\hline $08: 13-08: 20$ & & 08:08-08:11 & 08:08 & 08:07-08:18 & C2.6 08:06 08:13 & \\
\hline$(+2+12$ & & 08:15 & faint II? & $1.4280^{\circ}$ & & \\
\hline$-2+5)$ & & No $L$ waves & 08:20@40 & & & \\
\hline 1999 Feb. 20 & & III $>14 \mathrm{MHz}$ & III 20-70 & I G & N19W58 SF 09:13 & 27232160 \\
\hline $09: 14-09: 24$ & $\sim 1-200$ & 09:14 & & $09: 13$ & C1.1 09:11 09:18 & \\
\hline$(0+10)$ & & No L; BS & & $1.2255^{\circ}$ & & \\
\hline $09: 30-09: 40$ & $\sim 1-300$ & $09: 31$ & III 20-70 & I G 09:32 & & 27232160 \\
\hline$(-1+9)$ & & No L; BS & & $1.2255^{\circ}$ & & \\
\hline 09:55-10:05 & $\sim 1-300$ & III (faint) & No data & III? & N18W58 SF 09:52 & 27232160 \\
\hline$(0+10)$ & & 09:55 & & $09: 52-09: 55$ & N20W60 SF 10:00 & \\
\hline & & Poss. L; BS & & $1.4285^{\circ}$ & $\begin{array}{l}\text { C1.7 09:51 09:54 } \\
\text { C1.7 09:58 10:01 }\end{array}$ & \\
\hline $15: 15-15: 17$ & $\sim 1-300$ & III $>14 \mathrm{MHz}$ & No data & III & S17W71 SF 15:14 & None \\
\hline$(-4+4)$ & & $15: 13-15: 19$ & & $15: 12-15: 23$ & C4.2 15:11 15:19 & At W limb \\
\hline & & L waves; BS & & $1.2270^{\circ}$ & & \\
\hline 1999 May 27 & $\sim 3-\geq 500$ & III $>14 \mathrm{MHz}$ & III 20-70 & III & None Reported & Н 3601691 \\
\hline $10: 50-10: 55$ & & 10:39-11:09 & II $20-70$ & $10: 50$ & No event & \\
\hline$(-19+16)$ & & $\begin{array}{c}\text { IV/II 10:55@14 } \\
\text { Possible L }\end{array}$ & $\begin{array}{r}10: 46-10: 51 \\
\geq 11: 02\end{array}$ & $1.5288^{\circ}$ & & \\
\hline 1999 Jun. 29 & $\sim 1-300$ & III $>14 \mathrm{MHz}$ & III 20-70 & I and III & $\mathrm{N} 21 \mathrm{~W} 45$ 1F 12:40 E & None \\
\hline $12: 35-12: 45$ & & $12: 36-12: 42$ & & $12: 39-12: 45$ & C2.8 12:40 12:47 & Reported \\
\hline$(-7+9)$ & & L waves & & $0.9290^{\circ}$ & & \\
\hline 2000 Feb. 18 & $\sim 3-300$ & III $>14 \mathrm{MHz}$ & III, II 70-20 & II & S16W78 SF 09:25 & 286118890 \\
\hline $09: 25-09: 32$ & & $09: 16-09: 24$ & $09: 20-09: 25$ & $09: 18-09: 24$ & C1.1 09:21 09:27 & \\
\hline$(+1+16)$ & & L waves & $09: 25-09: 52$ & $1.5285^{\circ}$ & & \\
\hline 2000 Mar. 6 & $\sim 30-300$ & III $>14 \mathrm{MHz}$ & III 20-70 & III 12:11 & (S12 E27 SF 11:50) & None \\
\hline $12: 16-12: 22$ & & $12: 11+$ storm & & I $12: 20$ & C4.3 11:51 12:02 & Reported \\
\hline$(+5+11)$ & & L waves & & $0.9275^{\circ}$ & & \\
\hline 2000 Apr. 19 & $\sim 30-100$ & III $>14 \mathrm{MHz}$ & No data & III & (N13E02 SF 12:17) & 28523543 \\
\hline $12: 29-12: 39$ & & $12: 30-12: 33$ & & $12: 29-12: 34$ & (N18E04 SF 12:32) & \\
\hline$(-4+9)$ & & No $L$ waves & & $0.4275^{\circ}$ & C1.2 11:54 12:34 & \\
\hline 2000 May 1 & $\sim 1-\geq 500$ & III $>14 \mathrm{MHz}$ & No data & III & N21 W51 10:24EIT & 323541360 \\
\hline $10: 14-10: 25$ & & $10: 17-10: 23$ & & $10: 18-10: 23$ & M1.1 10:16 10:27 & \\
\hline$(-9+8)$ & & L waves; BS & & $1.2305^{\circ}$ & & \\
\hline 2000 Jun. 23 & $\sim 3-\geq 500$ & III $>14 \mathrm{MHz}$ & III $20-70$ & III/DCIM & N23W73 1F 14:20 & $293>103847$ \\
\hline $14: 28-14: 34$ & & $14: 20-14: 28$ & $14: 20-14: 28$ & $14: 20-14: 32$ & M3.0 14:18 14:31 & \\
\hline \multirow[t]{2}{*}{$(0+14)$} & & II 14:40@14 & II 14:33@60 & $1.5295^{\circ}$ & & \\
\hline & & L waves; BS & & & & \\
\hline 2000 Aug. 22 & $\sim 30-100$ & III $>14 \mathrm{MHz}$ & III 20-70 & III & N15W38 SN 09:36 & 27354534 \\
\hline $09: 46-09: 52$ & & $09: 40-09: 43$ & & 09:40-09:48 & C2.7 09:39 09:49 & \\
\hline$(+3+12)$ & & $\mathrm{L}$ waves & & $0.8285^{\circ}$ & & \\
\hline 2000 Nov. 8 & $\sim 30-300$ & III $>14 \mathrm{MHz}$ & III 20-70 & III? & None reported & None \\
\hline $14: 32-14: 40$ & & $14: 21-14: 38$ & & $14: 28$ & C3.8 14:29 14:45 U & Reported \\
\hline$(-6+19)$ & & Possible L & & $1.6305^{\circ}$ & & \\
\hline 2001 Apr. 30 & $\sim 1-200$ & III $>14 \mathrm{MHz}$ & III & III & EIT 10:48 NW limb & 332411299 \\
\hline $10: 55-11: 03$ & & $10: 58$ & & $10: 58-11: 01$ & C2.2 10:55 11:08 & \\
\hline$(-3+5)$ & & L waves & & $1.1290^{\circ}$ & & \\
\hline
\end{tabular}


soft X-ray emission in the $(0.1-0.8) \mathrm{nm}$ range are given as measured by GOES and reported in Solar Geophys. Data or read from the data.

7. The "CME" column gives the central position angle (PA), width $(\Delta$; both in degrees) and linear projected speed $(v$, in $\left.\mathrm{km} \mathrm{s}^{-1}\right)$ of the associated coronal mass ejection from S. Yashiro's LASCO catalogue.

The events of Table 1 are mostly associated with metric type III emission. Due to the limited space in the Table, the spectrographic information contains only the most conspicuous features at radio wavelengths. Faint continua may also exist at metre waves, and the identification of a metric radio burst seen at $\mathrm{NRH}$ frequencies as "type III" may not adequately translate the complexity of the high frequency spectrum, e.g. the continuum at $327 \mathrm{MHz}$ and higher frequencies observed on 2000 May 1.

In four events no $\mathrm{H} \alpha$ patrol observations were reported. In one of them (2000 May 1, see Fig. 1c) EIT detected a compact brightening whose position is given in the table, and the Meudon heliograph actually saw the $\mathrm{H} \alpha$ flare. In two other cases (1996 Dec. 24 and 2001 Apr. 30) large-scale structural changes on the limb shown by the EIT daily movie point to flares in partially occulted active regions. The 1999 May 27 event was very faint at metre wavelengths and had no soft X-ray counterpart, presumably because the flaring active region was behind the limb. Due to the time coincidence and the duration at $164 \mathrm{MHz}$ we included this event in the sample associated with "short" metre wave emission, but longer lasting emission may have been occulted, so that the event might be closer to those of Sect. 2.5.

Most events of Table 1 were similar to 2000 May 1 in that the electron release window comprised the start of radio emission from metric to decametric wavelengths (11/18 events). In the first two events of 1999 Feb. 20 the metre wave association was uncertain. A type I noise storm started well before the electron release window and continued well afterwards. But increased burst activity occurred within the noise storm in time coincidence with type III bursts in the NDA frequency range and with the first two DH type III bursts, which suggests a relationship between the noise storm emitting electrons and the escaping beams. The NDA showed that these DH type III bursts started above $70 \mathrm{MHz}$. The third electron release at $10 \mathrm{UT}$ was accompanied by bright metric type III bursts that were seen by the NRH between 327 and $164 \mathrm{MHz}$. These bursts had a different source than the noise storm. The three electron releases were hence related with type III emission at metre wavelengths, and all were included in this table despite the uncertain NRH association of the first two.

In a few events a simple DH type III burst preceded the electron release window by several minutes: 1997 Nov. 23, 1998 Jul. 11 and Sep. 27, 2000 Mar. 6, Aug. 22, Nov. 8. Such delays are of the order of the expected systematic errors of the release window determination and may not be significant. We note, however, that in several cases distinct evidence of coronal energy release is found during the delayed release window, such as the rise of the soft X-ray flux (1997 Nov. 23, Sect. A.1) or late type III bursts (1998 Sep. 27). Metric noise storm bursts with faint DH type III counterparts were seen during the release window on 2000 Mar. 6, and faint DH type III bursts with no counterpart at NRH frequencies on 2000 Nov. 8. On 2000 Aug. 22, similar to 1997 Nov. 23, no DH type III burst was seen during the release window, but bursts at metre wavelengths. It seems that in most cases of Table 1 delayed electron release, provided it is of solar origin and not due to systematic errors, can be associated with metric and/or decametric radio emission that indicates new electron acceleration in the corona.

\subsubsection{Type II bursts during short duration radio events}

In one case (1996 Dec. 24) a metric type II burst occurred during the release window, together with a group of type III bursts. In another event (2000 Feb. 18, cf. Klassen et al. 2002) the type II burst was the only metre wave emission near the release window, but the electron release started several minutes after the metric type II burst, together with decametric type II emission (cf. Fig. 10 of Maia \& Pick 2004) and during a weak soft X-ray event that itself lagged the onset of the metre wave type II burst. Only during the events on 1999 May 27 and 2000 Jun. 23 was a DH type II burst reported in the WAVES event list at NASA-GSFC. As already stated, part of the radio emission may have been occulted in the May 1999 event. On 2000 June 23 the type II burst started near $60 \mathrm{MHz}$ shortly after the electron release window. A similar type II burst may have been observed on 1998 Sep. 27, but the spectral signature is faint, and late type III emission started well above the type II band.

\subsection{Near-relativistic electron releases during long duration radio events}

The context information on those electron releases which occurred with long duration metric radio emission is summarised in Table 2. It is readily visible from Col. 1 that in several cases the electron release window lags by up to several tens of minutes the earliest DH type III burst or the start of metric radio emission. Case studies of prompt and delayed events are presented in Appendix A.2. In order to illustrate the processes which may create such delays, we discuss the most conspicuous event in the following.

\subsubsection{September 12: Electron release with late metre wave sources and post flare loops}

The 2000 Sep. 12 event was associated with the eruption of a large quiescent filament, discussed in detail by Vršnak et al. (2003a,b) and Schuck et al. (2004). The electrons detected by Wind/3DP were released more than $40 \mathrm{~min}$ after the onset of a bright DH type III burst, which started itself several minutes after the metric radio emission (Fig. 2a).

The time histories in Fig. 2a show the first metric radio emission $(327,164 \mathrm{MHz})$ during the rise of the soft X-ray burst, with no counterpart at frequencies below $70 \mathrm{MHz}$. The $1 \mathrm{D}$ brightness plots display a moving radio source at $327 \mathrm{MHz}$ during this early phase of the event, accompanied by rather faint emission at $164 \mathrm{MHz}$. The moving radio source was shown by Vršnak et al. (2003a) to be part of a broadband 
Table 2. 3DP events related to prolonged metre-wave emission.

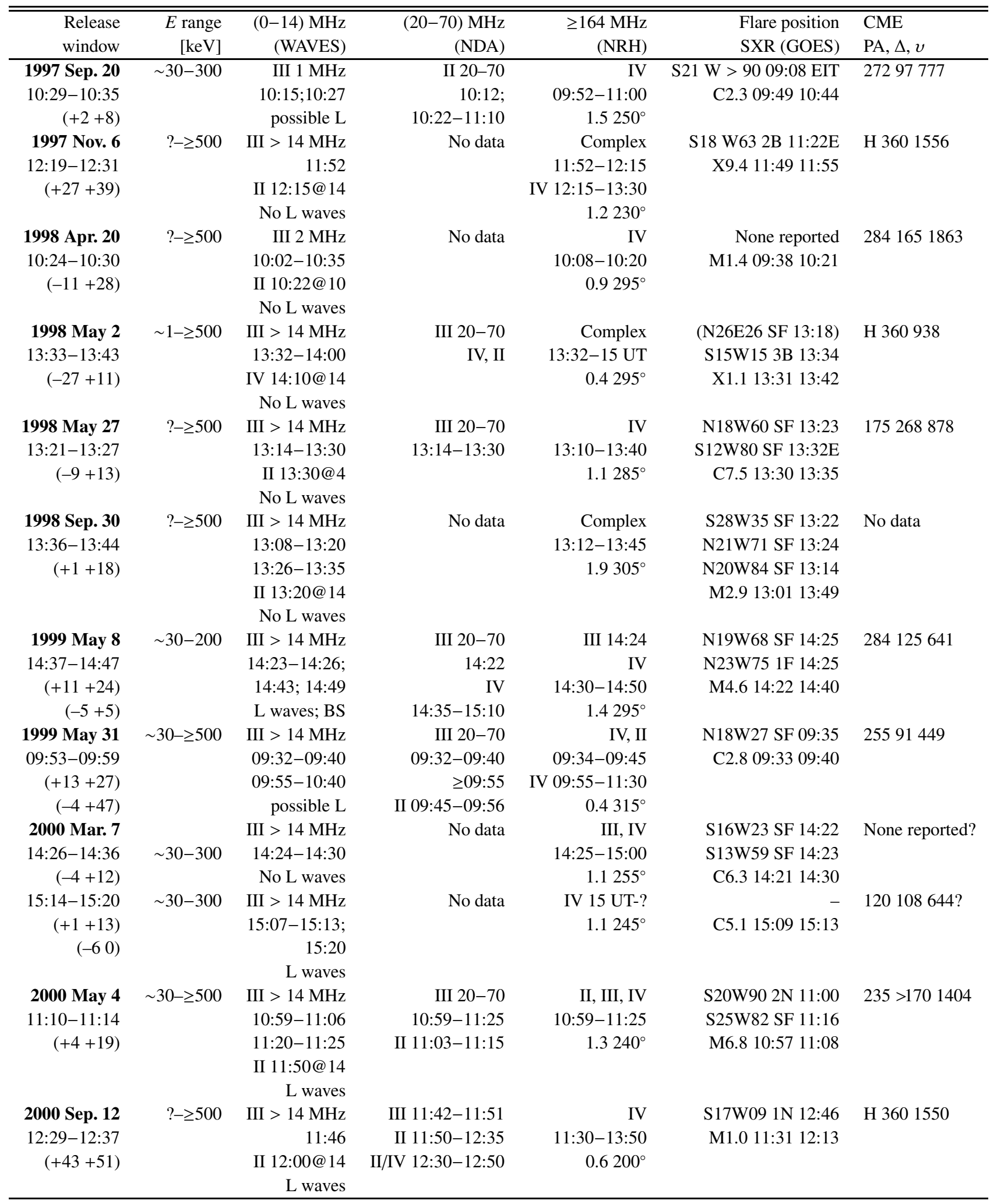

moving type IV burst probably located ahead of the rising filament. The first bright $164 \mathrm{MHz}$ emission occurred near the end of the moving type IV burst. It was followed in the (20-30) $\mathrm{MHz}$ range by a burst which gradually drifted towards lower frequencies, and seemed to be continued by slowly drifting narrow bands of type II emission in the (1-14) $\mathrm{MHz}$ range (12:00-12:30 UT).

A single bright DH type III burst appeared at frequencies between 30 and $14 \mathrm{MHz}$ during the metric continuum and the type II burst. At this time the emission at $327 \mathrm{MHz}$ rose in 

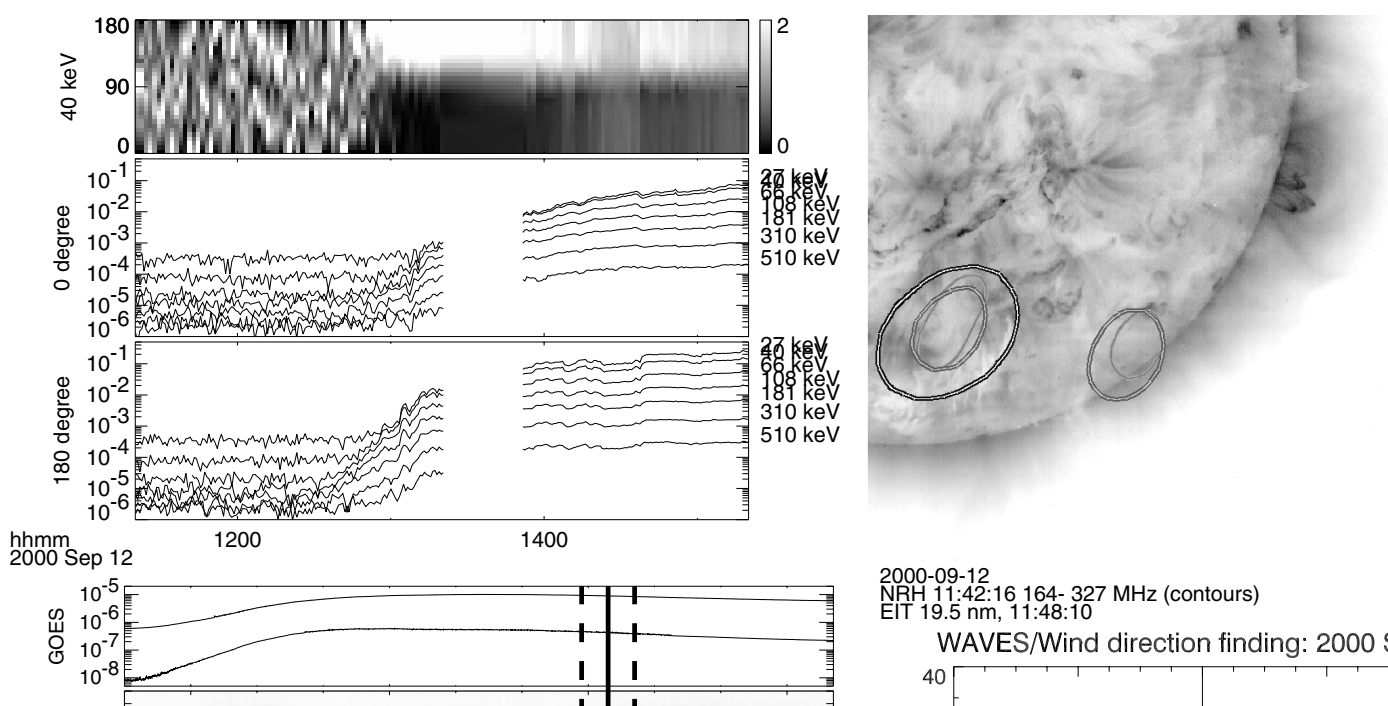

2000-09-12.
NRH 11:42:16 $164-327 \mathrm{MHz}$ (contours)
EIT 19.5 $\mathrm{nm}, 11: 48: 10$
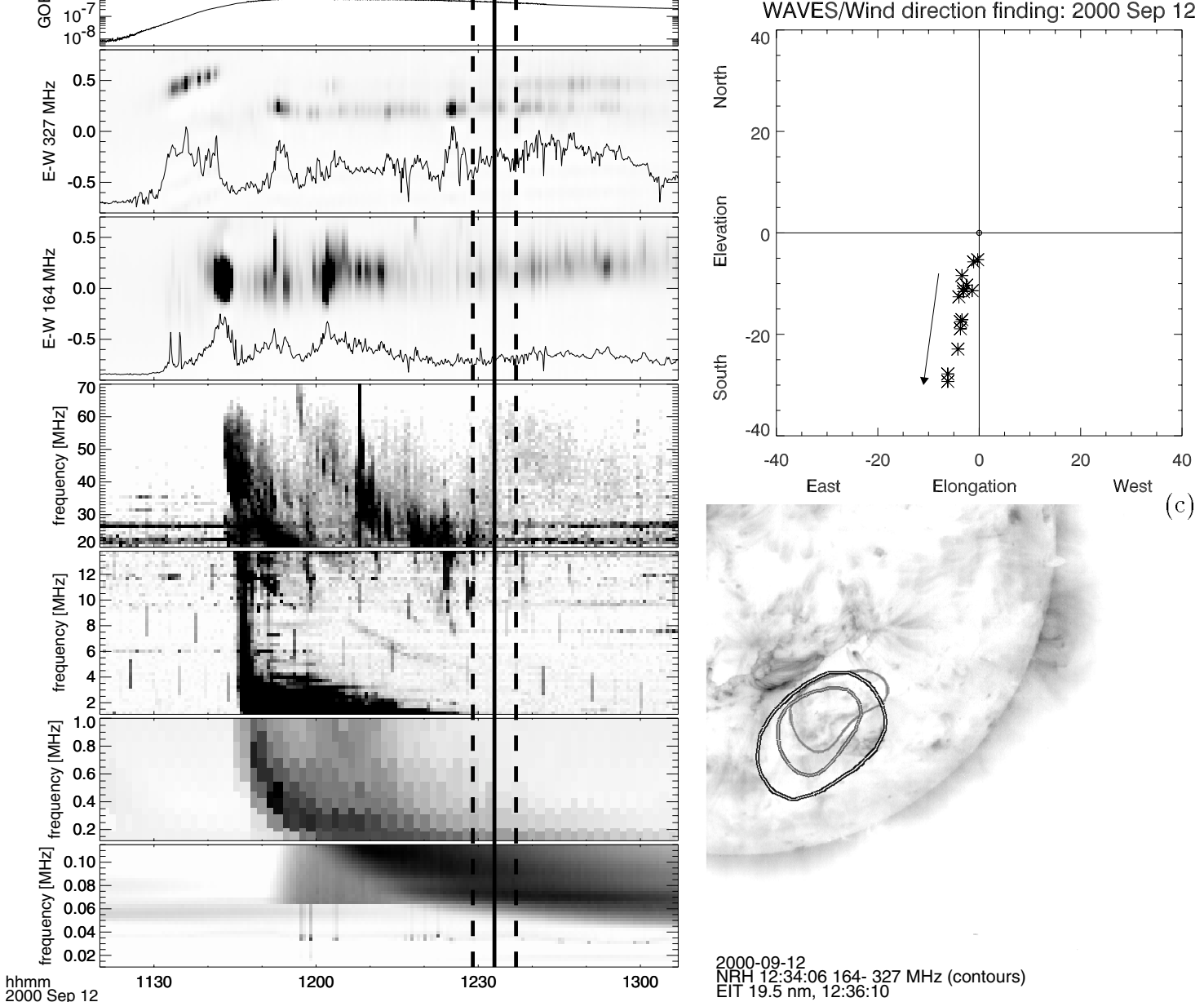

(a)

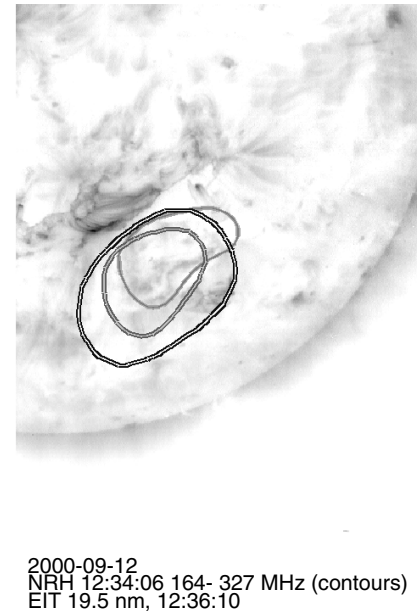

(c)

Fig. 2. 2000 September 12: a) time history of electrons detected at Wind/3DP (top) and of electromagnetic signatures. The uniform brightness increase just before $12 \mathrm{UT}$ in the TNR spectrum (bottom panel) is due to interference between different channels in the high-frequency detector band of TNR. (b), d)) Iso-intensity contours at half maximum of the radio emission on top of EIT images during the DH type III burst (b) and the electron release window (d), c)) centroids of the radio sources at frequencies between 940 and $176 \mathrm{kHz}$. The apparent radii of the radio sources increase from $20^{\circ}$ at the highest frequencies to about $40^{\circ}$ at the lowest. The arrow gives the direction from high to low frequencies.

a new source east of the moving one, which becomes clearly visible after 11:50 UT in the 1D brightness plot of Fig. 2a. Vršnak et al. (2003b) showed that at the same time the erupting filament changed morphology and separated into two different pieces, while underneath an $\mathrm{H} \alpha$ ribbon protruded over a coronal depletion seen by EIT/SoHO that had formed a few minutes earlier. These authors argued that magnetic reconnection of the rising arcade around the filament with the open field lines rooted in the newly formed coronal depletion allowed the electrons to escape to the high corona and interplanetary space. 
The metric radio emission that started with the DH type III burst was a type IV continuum ("statonary" type IV). It came from the easternmost source in the overlay on an EIT image in Fig. 2b. The source at the south-western limb was part of the moving type IV burst travelling ahead of the rising filament. The filament itself is no longer visible in the EIT image, but a pair of patchy flare ribbons is discerned just north of the stationary type IV source. The centroid positions of the hectometric-to-kilometric type III burst, inferred from the rotational modulation of the radio signal received by Wind/WAVES at frequencies below $1 \mathrm{MHz}$, are plotted in Fig. 2c. Each asterisk gives the position at one frequency. The arrow points in the direction of decreasing frequencies. The radio sources at all frequencies marginally comprise the ecliptic plane, but the centroids are the further away, the lower the frequency, i.e. the bigger the heliocentric distance. The projected trajectory of the type III emission points far southward, and extrapolates back to the southern location of the metric type IV radio sources. Although the raw centroid positions of these very large radio sources cannot be considered as an accurate tracer of interplanetary field lines (Reiner 2001), the observed southward trend of the positions with increasing heliocentric distance suggests that the bulk of the type III producing electron beams missed the spacecraft. So no near-relativistic electrons could be detected either by Wind/3DP with the DH type III burst. This is consistent with the fact that the low-frequency type III spectrum does not extend down to the plasma line measured at the spacecraft (Fig. 2a, bottom panel). The detection of Langmuir waves is not in conflict with this conclusion: their very early start, ten minutes after the onset of the type III emission at $14 \mathrm{MHz}$, would imply that they are produced by electrons with energies near $400 \mathrm{keV}$. Such electrons are not seen by the 3DP detectors at that time. The Langmuir waves detected by WAVES therefore cannot have been produced by the type III emitting electron beams.

The near-relativistic electrons attaining Wind/3DP started to be released (Fig. 2a) during the rise of a new broadband metric radio emission (stationary type IV) that is visible at $\mathrm{NRH}$ frequencies and, faintly, in the NDA spectrum. The rise of emission is mainly due to a new source just west of the former one. Both sources contributed to the type IV emission. At $164 \mathrm{MHz}$ they are not resolved, but the increasing importance of the western one is revealed by the apparent westward drift of the emission in the 1D brightness plot of Fig. 2a. The sources show up in Fig. 2d as a single elongated contour at each frequency. The bright structures in the EIT image just north of the radio sources are (post-) flare loops below the radio sources. The loop system continued to develop afterwards, attaining its final spatial extent about $50 \mathrm{~min}$ after the release window. No DH type III burst was observed by NDA or WAVES during the release window.

\subsubsection{Overview of the sample associated with long metric radio emission}

The 2000 Sep. 12 event shows delays between different types of electron signatures: decimetric-to-metric radio emission starts first, followed some minutes later by a DH type III burst that shows the first electrons escaping to interplanetary space. Electron acceleration proceeds over more than an hour in the low and middle corona, i.e. at heights $\lesssim 1 R_{\odot}$ above the photosphere, as shown by the brightening of new radio sources, and at shocks at greater heights, as shown by DH type II bursts. The near-relativistic electrons detected by Wind/3DP are released during the establishment of the new type IV source, which is suggestive of magnetic restructuring in the middle corona. No $\mathrm{DH}$ type III burst is seen during the release window.

Other events with a conspicuous delay between the release of near-relativistic electrons reaching Wind/3DP and the earliest radio signatures were 1997 Nov. 6 and 1998 Sep. 30. On 1997 Nov. 6 (see Krucker et al. 1999, for details) the electrons were released in the late decay phase of the brightest metric emission, together with a type IV continuum, but without a DH type III burst (see the spectrum in Fig. 3 of Reiner et al. 2000). On 1998 Sep. 30, the release window comprised a moving type IV burst (cf. detailed discussion in Appendix A.2.3). Delayed electron release occurred also at the time of a moving type IV burst on 2000 May 4.

On 1998 May 27 the electrons were released during a type IV continuum and a group of DH type III bursts. The release window contained the brightest of them. The release windows of the events 1999 May 8 and 31 and of the second event on 2000 Mar. 7 comprised the onset of type IV emission and DH type III bursts. In most of these events the type IV continuum occurred above regions where EIT later on observed the development of post flare loops at $19.5 \mathrm{~nm}$ wavelength.

Not all electron releases occurred after the onset of the metric radio event. The release windows on 1998 May 2 (cf. Appendix A.2.1 for details) and of the first event of 2000 Mar. 7 comprised the onset of metric radio emission as well as a DH type III burst. In summary, the release of near-relativistic electrons detected by Wind/3DP occurred during metric radio emission, be it near its onset or in the course of the radio event, mostly at the time of changes in the configuration or the brightness of the radio sources.

\subsubsection{Type II bursts during long duration radio events}

Type II bursts were detected in the majority of the events of Table 2, especially at frequencies below $70 \mathrm{MHz}$. They occurred most often at the time of long duration type IV emissions at higher frequencies. In two cases type II emission occurred during the release window without type IV counterpart at higher frequencies. Both were associated with activity which is partly occulted by the limb. The event of $1997 \mathrm{Sep} .20$ is discussed in detail in Appendix A.2.2. On 1998 Apr. 20 electrons were released onto field lines connected with the spacecraft together with a late group of DH type III bursts that started near $2 \mathrm{MHz}$, and with a faint and short type II burst detected by WAVES at a few MHz. Various aspects of this radio event were discussed in Gopalswamy (2000); Maia et al. (2000); Bastian et al. (2001); Klassen et al. (2002). The ambiguities in the association of the electron release window with the radio emissions were discussed in Klein et al. (2003a). 


\subsection{Summary of results}

\subsubsection{Near-relativistic electron events and DH type III bursts}

- All electron events observed by $3 D P$ in the selected observing period were associated with type III bursts or groups of bursts starting at frequencies $\geq 1 \mathrm{MHz}$. "Associated" here means that the type III emission occurred within some minutes or - in few cases - within some tens of minutes of the inferred time of near-relativistic electron release at the Sun.

- Most (35/40) of these type III bursts had starting frequencies above the WAVES upper detector limit at $14 \mathrm{MHz}$. All DH type III bursts accompanied by short metric emission (Table 1) started above $14 \mathrm{MHz}$, and 10/12 bursts associated with long duration metric radio emission (Table 2).

\subsubsection{Near-relativistic electron events, flares and CMEs}

- Nearly all events (exception: 1999 May 27) were accompanied by a soft X-ray burst, and most by a flare seen in $\mathrm{H} \alpha$ or EUV. For several long radio events in Table 2 two flares were reported to occur during the release window of the electrons, at angular distances of up to several tens of heliocentric degrees from each other. Events without flares were likely associated with activity behind the limb.

- CMEs were reported with 10 of the 15 events associated with short duration metric emission where LASCO observations were available (Table 1), and with 9/11 events associated with long duration radio emission (Table 2). The speeds and widths vary between the two categories: the average CME speed of the short duration events was $(745 \pm 434) \mathrm{km} \mathrm{s}^{-1}$, their average width $\left(62^{\circ} \pm 33^{\circ}\right)$, while the long duration events had faster $\left((1070 \pm 483) \mathrm{km} \mathrm{s}^{-1}\right)$ and broader $\left(210^{\circ} \pm 115^{\circ}\right)$ CMEs. Because of the ambiguity due to occultation, the 1999 May 27 event was not used in this evaluation. The great widths of the CMEs and the frequent occurrence of multiple flares during the long radio events suggest that these events are also characterised by a large coronal volume participating in the flare and CME.

\subsubsection{Near-relativistic electron events and impulsive electron signatures in the corona}

- The type III bursts observed by WAVES during short radio events start in the metre wave range. This means that the electron beams are accelerated in a plasma with ambient electron density above $10^{7} \mathrm{~cm}^{-3}$ (corresponding to type III emission at the harmonic of the plasma frequency starting above $60 \mathrm{MHz}$ ). In most cases the imaging observations at frequencies above $164 \mathrm{MHz}$ imply acceleration sites at heights $\lesssim 0.5 R_{\odot}$ above the photosphere and ambient electron densities $\gtrsim 10^{8} \mathrm{~cm}^{-3}$.

- Groups of metric type III bursts are usually observed. They give evidence for multiply impulsive electron acceleration in the corona. Imaging with arc minute spatial resolution often allows us to identify different magnetic flux tubes during one event that guide electrons to interplanetary space.

- The release window of electrons detected by Wind/3DP comprises distinct radio signatures of energetic electrons and energy release in the corona. These signatures may (12/18 events) or may not be among the first radio signatures of energetic electrons. Delays with respect to the first radio signatures mostly amount to a few minutes, i.e. are comparable with the systematic errors of release time determinations especially in weak events or events with high background. Nevertheless, in several cases the observations suggest that a delayed electron release may also reveal the delayed injection into the specific magnetic flux tube connected with the spacecraft.

- A type II burst was found in the vicinity of the release window in three or four events. But its presence is not a necessary condition for electron release to be delayed during these short duration events.

\subsubsection{Near-relativistic electron events with prolonged signatures of electrons in the corona}

- All long duration radio events comprised broadband metric continua with moving or stationary sources (or both) that belong to the broad group of type IV emissions, and most of them (9/12), but not all, were accompanied by DH type II bursts. DH type III bursts occurred as low frequency counterparts of impulsive metric emission that preceded the type IV bursts, or below the low frequency end of the type IV emission itself or on the low-frequency side of a type II spectrum.

- The relative timing of the release window and radio signatures is complex. Near-relativistic electrons reaching the spacecraft were occassionally released at the start of the radio event ( $2 / 12$ cases). In other events different signatures of energetic electrons were observed at different times: radio signatures of electrons in the corona seen at different frequencies may start at different times, DH type III bursts may start after the onset of the metric radio emission, and near-relativistic electrons reaching the spacecraft may be released after the start of the metric radio emission, after DH type III bursts, or both.

- Despite this complexity, there is a clear correlation between the release window and radio signatures of electrons in the corona. Even when the release window occurred in the course of the radio event, new electron populations in the corona were revealed by a new rise of the metric radio emission in a previously existing source or by a newly appearing source, which suggests the restructuring of the coronal magnetic fields at the heights of the metric radio emission.

- The non-detection of electrons at the spacecraft despite a clearly defined DH type III burst could in several cases be attributed to poor magnetic connection of the flux tubes guiding the electron beams responsible for the early type III bursts. This is consistent with the low detection rate of Langmuir waves during these events (Table 2). Conversely, 
near-relativistic electron release at the Sun need not be accompanied by a detectable DH type III burst.

\section{Discussion}

Using radio signatures from short metre waves to kilometre waves, we have probed the timing and propagation of energetic electrons from the middle corona, $\geq 0.1 R_{\odot}$ above the photosphere, to $1 \mathrm{AU}$ near the release times of near-relativistic electrons which eventually reached the Wind spacecraft. Three quarters of the electron events detected by Wind/3DP were accompanied by some type of metre wave radio emission, and all by DH type III bursts from electron beams travelling through the high corona and interplanetary space.

\subsection{The timing of different electron signatures}

Despite this global association the comparison between radio waves and in situ electron measurements reveals a complex behaviour. Most events show evidence that electrons are injected into different coronal structures at different times. As a rule, electrons reaching the Wind spacecraft are released at the time of a distinct signature of energetic electrons in the corona. In $16 / 30$ events the release of electrons detected at the spacecraft and of the beams emitting the DH type III bursts started at the onset of the metric radio emissions. In other events different signatures of energetic electrons were observed at different times. Hence delays between different signatures of energetic electrons are common and occur in a variety of ways, rather than being a characteristic of escaping electrons that are measured at the spacecraft. The fraction of events where the electrons reaching the spacecraft were released after the earliest radio signature depends on the accuracy with which the release window can be determined. Systematic errors of the release time determination cast doubt on the reality of delays of only a few minutes. The delays inferred from the present analysis are comparable to other studies (Krucker et al. 1999; Haggerty \& Roelof 2002; Cane 2003; Maia \& Pick 2004).

\subsection{Temporal and spatial complexity}

The radio emissions observed during the electron events under discussion unambiguously show the presence of non thermal electrons in the corona over durations ranging from a few minutes to several hours. Multiple metric type III bursts are produced by successive injections of electrons into open magnetic flux tubes and are most naturally interpreted as tracers of multiple electron accelerations. Broadband metre wave emission of long duration (type IV emission) is also a signature of repeated or long lasting electron acceleration, as indicated by the short lifetime of the radiating electrons - unless they are relativistic and by the repeated brightenings and new sources arising during a given event. We henceforth suppose that time-extended metric radio emission means time-extended acceleration, and not merely the time-extended presence, of electrons.

The observations presented in this work then show that some formerly known observational facts on coronal electron acceleration are relevant to the timing of electron release to interplanetary space:

- Since the acceleration of radio emitting electrons in the corona is a complex, multiply impulsive or quasicontinuous, process, a timing comparison between the release of electrons and only the earliest electromagnetic signatures of an event is inconclusive.

- Since electrons accelerated at different times during an event may be injected into different coronal structures, not all electrons accelerated during an event may have access to interplanetary space, and the electrons which do get access need not be released onto field lines connected with the spacecraft.

The second item implies that different timings of electrons detected in situ and electrons in the corona may be a problem of magnetic connection rather than acceleration. It also explains why there need not be an electron event detected at the spacecraft with each DH type III burst, since the type III radio waves are emitted into a broad cone around the beam direction (Hoang et al. 1997) and can therefore be seen even if the emitting electrons do not travel towards the observer. The association of nearly all electron events with CMEs, i.e. with the large-scale reconfiguration of the coronal magnetic field, furthermore suggests that the magnetic connection between acceleration sites in the corona and the foot of the spacecraft-connected interplanetary field line in the high corona may change in the course of an event.

Because all delayed electron releases, with the possible exception of a few weak events with short delays, occur together with distinct signatures of energetic electrons in the corona, we believe that the origin of the delayed electron releases is to be searched in the interplay between time-extended acceleration of electrons and their injection into different magnetic structures, not in the propagation of electrons in interplanetary space as suggested by Cane (2003).

\subsection{Near-relativistic electron releases and type III bursts}

Although electron releases to the high corona and interplanetary space are usually accompanied by DH type III bursts, there are noticeable exceptions. Type III emission is an indirect process, where electron beams excite Langmuir waves which couple to electromagnetic waves. There are several possible reasons why type III emission is weak or not observed during electron release in the corona. One is that while type III emission is well observed to be generated in magnetically quiet structures (Buttighoffer 1998), the electrons released in the aftermath of a CME travel through a highly disturbed corona and may not be able to form a sufficiently collimated beam. Such a scenario was proposed by Reiner \& Kaiser (1999) to explain the peculiar low-frequency ( $\leqslant 7 \mathrm{MHz}$ ) features of come complex DH type III groups. Furthermore, the model calculations of Raoult et al. (1990) give evidence that while the formation of a positive slope in the distribution function is a natural process during very short injections, where fast electrons outpace the 
slower ones, the continuous replenishment of electrons in timeextended injections can overcome this effect so that favourable conditions for Langmuir wave excitation may exist only at the beginning of these events. Both arguments explain why delayed electron releases accompanied by long duration metre wave events and complex CMEs may not be accompanied by DH type III bursts.

\subsection{Candidate processes for late acceleration: Shock waves and post-eruptive reconnection}

Two candidate processes have been discussed for delayed particle acceleration during a flare and CME: extended coronal shock waves (Krucker et al. 1999; Reames 1999; Simnett et al. 2002) and particle acceleration in post-eruptive reconnection (Cliver et al. 1986; Litvinenko \& Somov 1995; Akimov et al. 1996; Klein \& Trottet 2001). Evidence for both can be drawn from the radio emissions associated with the Wind/3DP electron events. We note, however, that there is no hint that shock acceleration actually explains delays, since prompt and delayed releases are both observed together with fast CMEs. Neither can the acceleration of electrons producing the long lasting metre wave emission be explained by a coronal shock, since the shock travels through the metre wave source in a time lapse much shorter than the event duration. The electrons it may accelerate have lifetimes of minutes at most. Hence even when a type II burst demonstrates that a shock wave exists, a different mechanism of long lasting acceleration must operate in the corona.

In numerous cases late electron release is associated with moving radio sources or late type IV emission in regions where post flare loops are subsequently observed. This points to the importance of post-eruptive magnetic reconnection as the source of near-relativistic electrons in space. Reconnection is required to partially detach the moving radio sources from the coronal magnetic field structure (see, e.g., the scenario of flux rope formation in Gosling et al. 1995), and is also the key process in the formation of flare loops. Since well-observed events provide evidence that a new episode of coronal electron acceleration, revealed by the brightening of a new radio source or of a previously existing one, occurred at the time of a delayed electron release, we conclude that in many cases the electrons detected in situ are accelerated together with those emitting the metre waves, and not at the shock which provides the type II emission. This does not mean that all events where evidence for post-eruptive reconnection exists produce energetic particles in space. Kahler et al. (2000) showed a sample of cases where the formation of large-scale arcades, which is probably due to reconnection in the post-CME region, did not provide solar energetic particle events. But since the arcades which were not associated with particle events (protons $\sim 20 \mathrm{MeV}$ ) had only weak or no radio signatures of electron acceleration in the corona, their conclusion is not in conflict with ours.

Our findings do not exclude that electrons are also accelerated at CME shocks. But while in a few events the association between electron release and type II burst emission argues in favour of shock acceleration, more definite timing relationships exist in the majority of events between delayed electron releases and the acceleration of radio emitting electrons at lower heights than the shock. We note that the most convincing cases for a temporal association between a type II burst and the release of energetic electrons are found in events where a significant fraction of the radio emission may have been occulted, because it occurred behind the solar limb (1997 Sep. 20, 1998 Apr. 20). These cases are reminiscent of the finding by Hucke et al. (1992) that relativistic electron events associated with nominally poorly connected flares are accompanied by DH type II emission. This may mean that type II shock waves do contribute to the acceleration of electron events, especially when there is no magnetic connection between the spacecraft and the coronal acceleration sites behind the CME. But the number of two events with a pure type II radio counterpart during the release window is insufficient for a firm statement.

The above conclusions are based on the three quarters of solar energetic electron events which were associated with metric radio emission. Why are there electron events without associated metric radio emission? One answer is that there is no reason that the lowest frequency where regular solar imaging observations are avavailable, i.e. $164 \mathrm{MHz}$, is a natural frontier and that no electron acceleration can occur at greater altitudes. The fact that most electron events with no counterpart at $164 \mathrm{MHz}$ are accompanied by DH type III bursts which start above $14 \mathrm{MHz}$ suggests a coronal acceleration site at greater heights than those accessible to metre wave imaging. Further evidence for this interpretation was provided by Maia \& Pick (2004). There are also more events associated with type II bursts in Table A.1, which may increase the number of cases where type II emission from CME-driven shock waves is the most probable association with the electron release. The most convincing case for acceleration at large-scale coronal perturbations, related with EIT waves and shocks, remains the eastern hemisphere event of 1997 April 7, which has a well-defined correspondence at metre wavelengths - with sources east of central meridian - and in DH type III bursts. The detailed radio data give no new elements for or against the interpretation proposed by Krucker et al. (1999), which supports the importance of shock acceleration in poorly connected events.

\section{Conclusions}

The evolution of metric-to-kilometric radio signatures provides evidence that electrons may be accelerated over extended time periods (several minutes to a few hours) at heights below about $0.5 R_{\odot}$ above the photosphere, and released into a range of closed and open magnetic structures. This complexity is able to explain a broad variety of timings between different electron signatures. The relative timing of electrons detected in situ and radio signatures in the corona depends on the magnetic connection of the spacecraft with the coronal structures into which the electrons are released. Since there is no a priori reason that the spacecraft is connected with the specific flux tube into which the first accelerated electrons are injected, the observed delays between the release of electrons detected in situ and the earliest metric signature of coronal acceleration or with type III bursts signalling the escape of electrons to interplanetary space 
are to be expected. Two candidate processes have been invoked to explain electron acceleration after the impulsive flare phase. While shock acceleration is plausible in some of the studied events, time-extended acceleration due to the restructuring of the solar corona in the aftermath of CMEs is emphasised by the majority.

Acknowledgements. Research with Wind/3DP is funded by NASA grant NAG5-6928 at Berkeley. The Wind/WAVES investigation is a collaboration of the Observatoire de Paris, NASA Goddard Space Flight Center and the University of Minnesota. The Nançay Radio Observatory is funded by the French Ministry of Education, the CNRS and the Région Centre. Part of this research was supported by the France Berkeley Fund. This work benefitted from the data of EIT and LASCO aboard SoHO, Yohkoh/SXT, the radio experiment WAVES aboard Wind, the radio spectrographs in Moscow (IZMIRAN), Potsdam-Tremsdorf, Nançay, and Zurich, through the respective web-pages. GOES data were provided through the Solar Data Analysis Center at NASA Goddard Space Flight Center. Extensive use was made of the SoHO/LASCO catalogue of CMEs at Goddard Space Flight Center and the Catholic University of America. SoHO is a project of international cooperation between ESA and NASA. We acknowledge H. Aurass (Potsdam), A. Lecacheux and P. Zarka (Meudon) for providing detailed data from the Tremsdorf and Nançay radio spectrographs, H. Hudson and D. Maia for helpful discussions. The referee is thanked for his/her most helpful detailed and constructive comments on the manuscript.

\section{References}

Akimov, V. V., Ambrož, P., Belov, A. V., et al. 1996, Sol. Phys., 166, 107

Bagalá, L. G., Stenborg, G., Schwenn, R., \& Haerendel, G. 2001, JGR, 106, 25239

Bai, T., \& Ramaty, R. 1979, ApJ, 227, 1072

Bastian, T. S., Pick, M., Kerdraon, A., Maia, D., \& Vourlidas, A. 2001, ApJ, 558, L65

Bougeret, J.-L., Kaiser, M. L., Kellogg, P. J., et al. 1995, Space Sci. Rev., 71, 231

Burlaga, L. F., Skoug, R. M., Smith, C. W., et al. 2001, JGR, 106, 20957

Buttighoffer, A. 1998, A\&A, 335, 295

Cane, H. V. 2003, ApJ, 598, 1403

Cliver, E. W., Dennis, B. R., Kiplinger, A. L., et al. 1986, ApJ, 305, 920

Delaboudinière, J. P., Artzner, G. E., Brunaud, J., et al. 1995, Sol. Phys., 162, 291

Gopalswamy, N. 2000, in AGU Monograph, Radio Astronomy at Long Wavelengths, ed. R. Stone, K. Weiler, M. Goldstein, \& J.-L. Bougeret, 119, 123

Gosling, J. T., Birn, J., \& Hesse, M. 1995, GRL, 22, 869

Haggerty, D. K., \& Roelof, E. C. 2002, ApJ, 579, 841

Hoang, S., Dulk, G., \& Leblanc, Y. 1994, A\&A, 289, 957

Hoang, S., Poquérusse, M., \& Bougeret, J.-L. 1997, Sol. Phys., 172, 307

Hucke, S., Kallenrode, M. B., \& Wibberenz, G. 1992, Sol. Phys., 142, 143

Hudson, H. S. 1978, ApJ, 224, 235

Kahler, S. W., McAllister, A. H., \& Cane, H. V. 2000, ApJ, 533, 1063

Kallenrode, M. B., \& Wibberenz, G. 1990, in Proc. 21st ICRC, 5, 229

Kerdraon, A., \& Delouis, J. 1997, in Lecture Notes in Physics, Coronal Physics from Radio and Space Observations, ed. G. Trottet (Springer), 483, 192
Klassen, A., Bothmer, V., Mann, G., et al. 2002, A\&A, 385, 1078

Klassen, A., Pohjolainen, S., \& Klein, K.-L. 2003, Sol. Phys., 218, 197

Klein, K.-L., \& Trottet, G. 2001, Space Science Rev., 95, 215

Klein, K.-L., Khan, J. I., Vilmer, N., Delouis, J., \& Aurass, H. 1999, A\&A, 346, L53

Klein, K.-L., Krucker, S., \& Trottet, G. 2003a, Adv. Space Res., 32, 2521

Klein, K.-L., Schwartz, R. A., McTiernan, J. M., et al. 2003b, A\&A, 409, 317

Kocharov, L. G., Kovaltsov, G. A., Kocharov, G. E., et al. 1994, Sol. Phys., 150, 267

Krucker, S., Larson, D. E., Lin, R. P., \& Thompson, B. J. 1999, ApJ, 519,864

Leblanc, Y., Dulk, G. A., Cairns, I. H., \& Bougeret, J. L. 2000, JGR, 105,18215

Lecacheux, A. 2000, in AGU Monograph, Radio Astronomy at Long Wavelengths, ed. R. Stone, K. Weiler, M. Goldstein, \& J.-L. Bougeret, 119, 321

Lin, R. P., Levedahl, W. K., Lotko, W., Gurnett, D. A., \& Scarf, F. L. 1986, ApJ, 308, 954

Lin, R. P., Anderson, K. A., Ashford, S., et al. 1995, Space Sci. Rev., 71,125

Litvinenko, Y. E., \& Somov, B. V. 1995, Sol. Phys., 158, 317

Maia, D., \& Pick, M. 2004, ApJ, 609, 1082

Maia, D., Pick, M., Vourlidas, A., \& Howard, R. 2000, ApJ, 528, L49

Manning, R., \& Fainberg, J. 1980, Space Science Instrumentation, 5, 161

McKenzie, D. E. 2000, Sol. Phys., 195, 381

McLean, D., \& Labrum, N. 1985, Solar radiophysics: studies of emission from the sun at metre wavelengths (Cambridge University Press)

Paesold, G., Benz, A. O., Klein, K.-L., \& Vilmer, N. 2001, A\&A, 371, 333

Pick, M. 1986, Sol. Phys., 104, 19

Pick, M., \& Ji, S. 1986, Sol. Phys., 107, 159

Pick, M., Maia, D., Wang, S., Lecacheux, A., \& Hawkins, S. 2003, Adv. Space Res., 32, 2527

Pohjolainen, S., Maia, D., Pick, M., et al. 2001, ApJ, 556, 421

Poquérusse, M., Hoang, S., Bougeret, J.-L., \& Moncuquet, M. 1996, in Solar Wind Eight, ed. D. Winterhalter, J. Gosling, S. Habbal, W. Kurth, \& M. Neugebauer (Am. Inst. Phys.), 62

Raoult, A., Mangeney, A., \& Vlahos, L. 1990, A\&A, 233, 229

Reames, D. V. 1999, Space Science Rev., 90, 413

Reiner, M. J. 2001, Spa. Sci. Rev., 97, 126

Reiner, M. J., \& Kaiser, M. L. 1999, GRL, 26, 397

Reiner, M. J., Karlický, M., Jiřička, K., et al. 2000, ApJ, 530, 1049

Schuck, P. W., Chen, J., Schwartz, I. B., \& Yurchyshyn, V. 2004, ApJ, 610, L133

Simnett, G. M., Roelof, E. C., \& Haggerty, D. K. 2002, ApJ, 579, 854

Torsti, J., Riihonen, E., \& Kocharov, L. 2004, ApJ, 531, L75

Trottet, G. 1986, Sol. Phys., 104, 145

Tsuneta, S., Acton, L., Bruner, M., et al. 1991, Sol. Phys., 136, 37

Vršnak, B., Klein, K.-L., Warmuth, A., Otruba, W., \& Skender, M. 2003a, Sol. Phys., 214, 325

Vršnak, B., Warmuth, A., Maričić, D., Otruba, W., \& Ruždjak, V. 2003b, Sol. Phys., 217, 187

Wang, T., Yan, Y., Wang, J., Kurokawa, H., \& Shibata, K. 2002, ApJ, 572,580

Warmuth, A., Hanslmeier, A., Messerotti, M., et al. 2000, Sol. Phys., 194,103 
K.-L. Klein et al.: Coronal phenomena at the release of solar energetic electron events, Online Material $p 1$

\section{Online Material}


K.-L. Klein et al.: Coronal phenomena at the release of solar energetic electron events, Online Material p 2

\section{Appendix A: Case studies}

\section{A.1. 1997 November 23: A short duration radio event with delayed electron release}

On 1997 Nov. 23 the electron release started about $10 \mathrm{~min}$ after a simple DH type III burst that was accompanied by metric type III radio emission (Fig. A.1a). The metric radio source was associated with a flare and a faint soft X-ray burst in the north-western quadrant (Fig. A.1b, top). Remarkably, the soft X-ray emission rose after the DH type III burst, but during the electron release window. The metric radio emission, which was brightest during the DH type III burst, continued afterwards at two positions, respectively east and west of the former type III source in the 1D brightness time history at $164 \mathrm{MHz}$ (Fig. A.1a). The change of the source configuration took place during the electron release window. The bottom panel of Fig. A.1b shows that both sources are connected with the same active region, but outline two diverging magnetic structures. The south-eastern one seems to be closer to the ecliptic plane, i.e. better connected to the spacecraft, than the type III source. Further evidence that the type III emitting electron beams did not reach the spacecraft stems from the lowfrequency spectrum at the bottom of Fig. A.1a. It shows no evidence that the type III emission actually approached the plasma frequency at the spacecraft, which is outlined by many shortlived bursts of Langmuir waves detected already well before this type III burst.

\section{A.2. Long duration events}

\section{A.2.1. 1998 May 2: Prompt electron release during a long duration radio event}

The near-relativistic electrons detected by Wind/3DP on 1998 May 2 were released during the first minutes of a complex and long duration radio event. The radio emission started at metre waves (e.g., 327 and $164 \mathrm{MHz}$ in Fig. A.2a). A few minutes later, at the time of the brightest metre wave emission during the release window, a group of DH type III bursts followed, extending from at least $70 \mathrm{MHz}$ down to some tens of $\mathrm{kHz}$. Figure A.2b shows snapshot maps of the radio and EUV emission. The flare, indicated by the black bar (due to saturation of the detector), occurred at moderate western longitudes, but had manifestations all over the solar disk (see Warmuth et al. 2000; Pohjolainen et al. 2001, for a detailed presentation of the optical, EUV, and radio imaging observations). Sources before the DH type III burst (top panel in Fig. A.2b) projected to the north and north-east of the flaring active region. The bright emission during the release window (bottom panel of Fig. A.2b) had sources extending north-westward from the flare. The Earth was in a magnetic cloud during this event (Burlaga et al. 2001; Torsti et al. 2004). Torsti et al. report that proton beams arrived along the local magnetic field which had a strong component out of the ecliptic plane and a slight east-west orientation. The peculiar structure of the magnetic field makes it impossible to draw significant conclusions on the electrons' access to interplanetary space from a comparison of the radio source locations and coronal magnetic field extrapolations (see Wang et al.
2002). But the broad range of directions into which electrons are injected in the corona during the release window suggests that the electrons arrived promptly at the spacecraft because they found access to the magnetic cloud right from the beginning of the coronal acceleration.

The coincident timing does not necessarily imply that the electrons detected at Wind/3DP were the high-energy part of the population emitting the DH type III burst. The WAVES TNR spectrum at the bottom of Fig. A.2a suggests that the burst did not reach the plasma line, which is shown by the faint grey line near $25 \mathrm{kHz}$, and that no Langmuir waves were detected. The type III emission was too complex to infer the source positions in interplanetary space through the direction finding technique. But it is readily visible in the top panel of Fig. A.2b and in Pohjolainen et al. (2001) that much of the metric radio emission also came from north and north-east of the flare site. The type III emitting electron beams may not have been intercepted by Wind.

Type II emission from shock-accelerated electrons during this event was discussed by Leblanc et al. (2000) and Reiner et al. (2000). From the sensitive NDA observations the former authors identified the onset of the type II burst at $45 \mathrm{MHz}$ and 13:44 UT. The type II burst was closely related with the long lasting decimetric-to-decametric type IV continuum, and started very shortly after the electron release window. The (20-70) MHz spectrum in Fig. A.2a shows that type III bursts occur during the type II emission, but they start at higher frequencies.

\section{A.2.2. 1997 September 20: Electron release associated with a type II burst}

The electron event detected by Wind/3DP (top panel of Fig. A.3) is weak, but clearly beamed. The parent flare was partly occulted. At 09:08 UT EIT observed the rise of a prominence above the south-western limb, together with the expansion of pre-existing overlying loops. At this latitude, active region NOAA 8084 had crossed the limb three days before. The bright tops of (post-) flare loops emerged from behind the limb since 10:50 UT at the latitude where the prominence had been seen to rise in the 09:08 UT image, and subsequently rose and spread in latitude during several hours.

Weak metre wave emission was observed by the NRH since about 09:42 at $327 \mathrm{MHz}$, and 09:50 UT at $164 \mathrm{MHz}$, i.e. well before the release window (Fig. A.3). Faint emission at $164 \mathrm{MHz}$ persisted during the release window, as shown by the whole Sun flux density. There is no report from patrol observations, presumably because the emission was very weak. The metric radio sources projected onto the region where the prominence erupted earlier, and where flare loops rose later. The emission occurred while the pre-event loop system seen by EIT pursued its expansion, and probably overlapped with the early formation of flare loops which still were occulted by the limb at the time of the metric radio emission. The faintness of the radio emission may indicate that the source was located well behind the plane of the sky, and that its emission was absorbed by foreground plasma. This would imply a source loca- 
K.-L. Klein et al.: Coronal phenomena at the release of solar energetic electron events, Online Material $p 3$
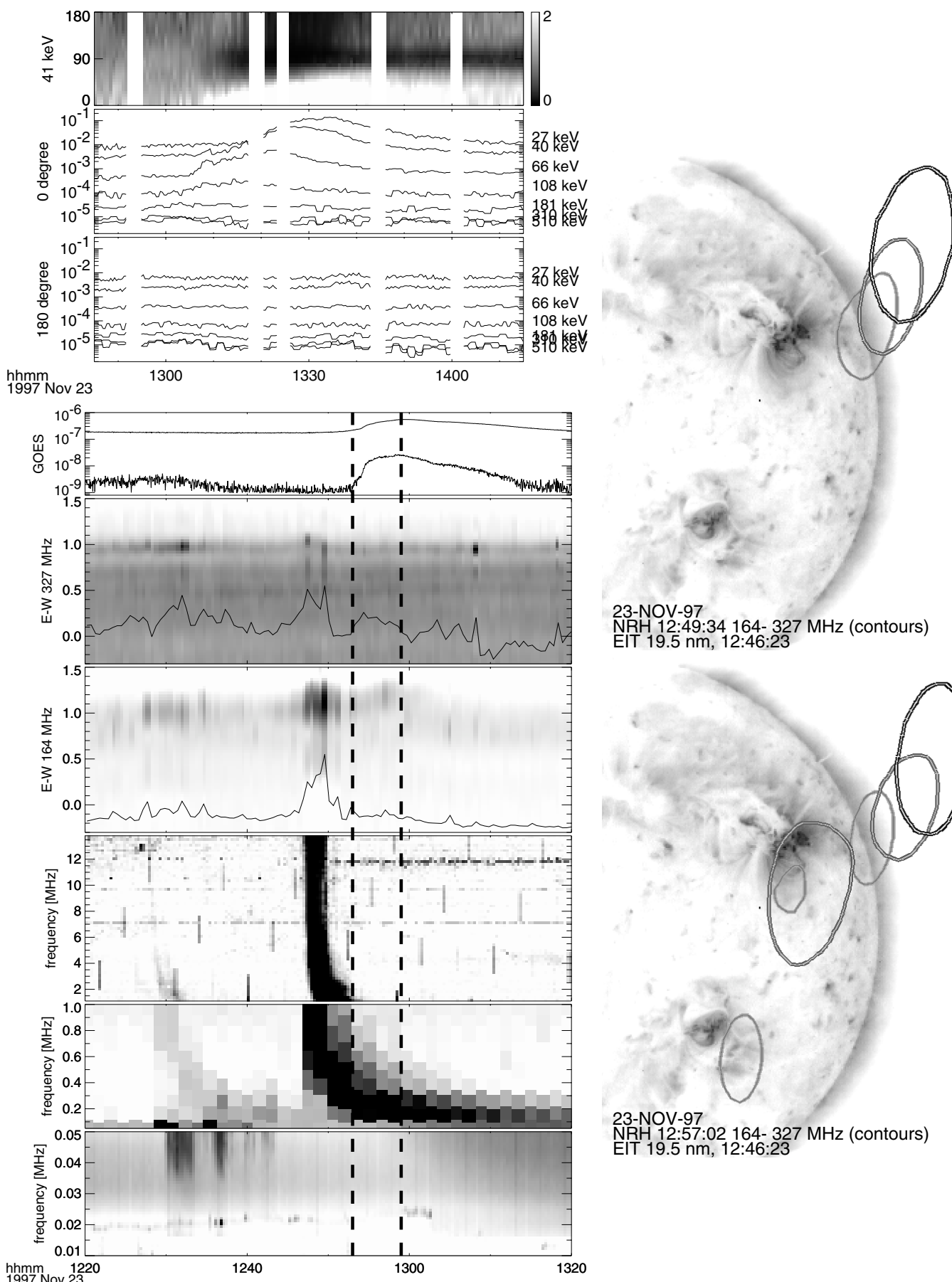

(a)

(b)

Fig. A.1. 1997 November 23: a) time histories of the electrons detected by Wind/3DP (top), comparison with the electromagnetic emissions (bottom). b) Metric radio sources during the DH type III burst (top) and the electron release window (bottom). Cf. Fig. 1. The contour above the active region in the south-western quadrant (bottom panel) outlines a noise storm with no specific relation to the event under discussion.

tion far from the nominal Earth-connected interplanetary field line. There is in any case no change in this emission that indicates a fresh coronal electron injection during the release window of the electrons detected at Wind.

The dominant feature of the spectrum between 75 and $25 \mathrm{MHz}$ in Fig. A.3 is a type II burst from a coronal shock that started about $30 \mathrm{~min}$ after the metre wave emission and just before the release window, together with a DH type III burst observed by WAVES at frequencies below a few MHz. The tim- ing of the release of the electrons detected at Wind/3DP points to a connection with the coronal shock wave.

\section{A.2.3. 1998 September 30: Electron release with moving metre wave sources}

The 1998 Sep. 30 event had a time history with multiple delays between different signatures of energetic electrons (Table 2, 
K.-L. Klein et al.: Coronal phenomena at the release of solar energetic electron events, Online Material p 4
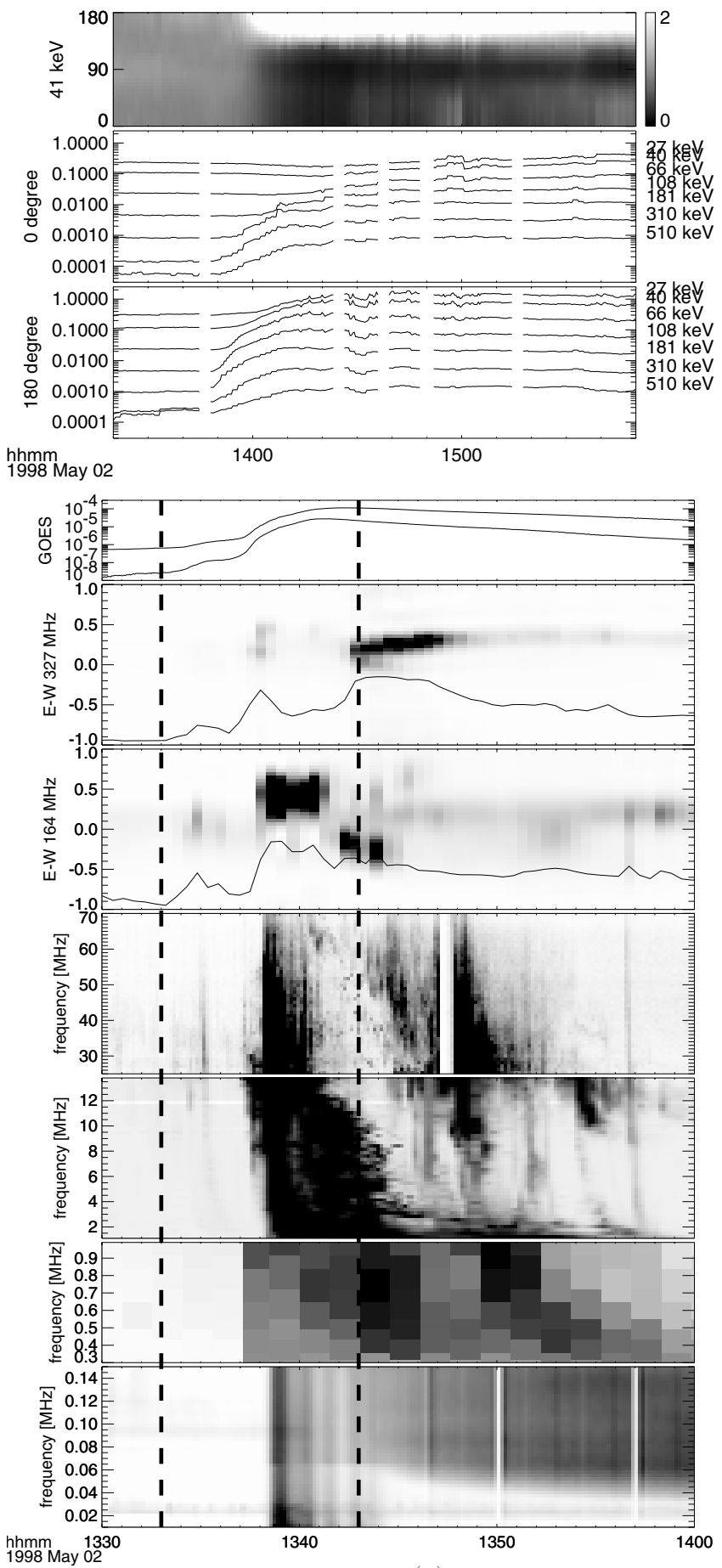

(a)

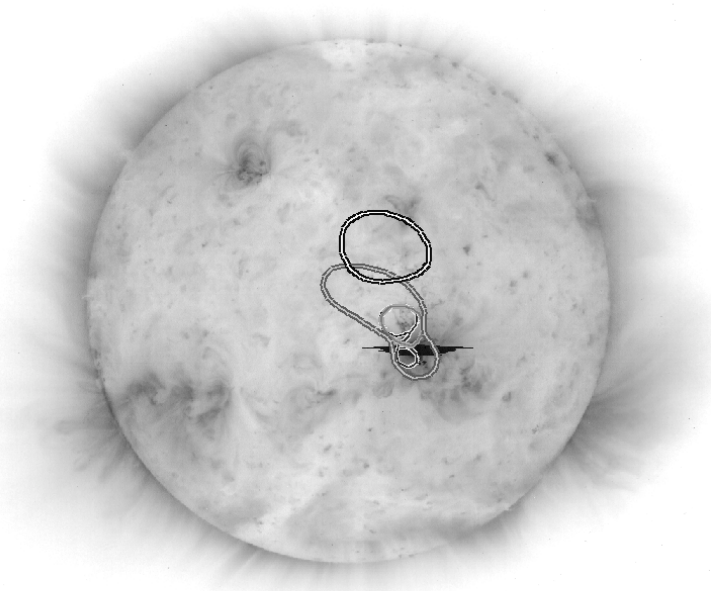

02-MAY-98 NRH 13:34:49 164- $432 \mathrm{MHz}$ (contours) EIT $19.5 \mathrm{~nm}, 13: 42: 05$

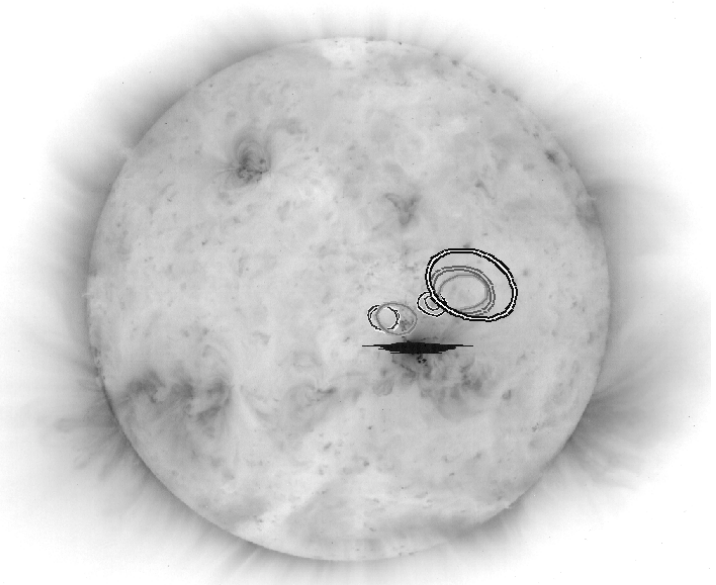

02-MAY-98 NRH 13:38:01 164- $432 \mathrm{MHz}$ (contours) EIT $19.5 \mathrm{~nm}, 13: 42: 05$

Fig. A.2. 1998 May 2: a) time histories of the electrons detected by Wind/3DP (top), comparison with the electromagnetic emissions (bottom). The vertical line near the onset of the type III burst in the WAVES TNR spectrum $(\sim 13: 38$; bottom panel $)$ is due to interference in the instrument. b) Metric radio sources before (top) and during (bottom) the DH type III bursts. Cf. Fig. 1.

Fig. A.4): (1) the electrons detected by Wind/3DP were released well after bright DH type III emission; (2) the bright DH type III emission occurred more than 10 min after the first radio signatures of electron acceleration in the corona; (3) the metric radio ermission started the later, the lower the frequency.

The dynamic metre wave spectrum (see Reiner et al. 2000, their Fig. 2) shows as the earliest signature of energetic electrons in the corona a broadband continuum whose low-frequency border gradually drifted to lower frequencies between its start (13:12 UT) and the onset of the bright DH type III bursts near 13:25 UT. The time histories of the 1D brightness distributions at 327 and $164 \mathrm{MHz}$ in Fig. A.4a also display this delay. In projection onto the solar east-west and north-south directions, the sources at $327 \mathrm{MHz}$ shifted systematically westward, while spreading in the north-south direction. The gradual drift of the frequency spectrum and the source 
K.-L. Klein et al.: Coronal phenomena at the release of solar energetic electron events, Online Material $p 5$
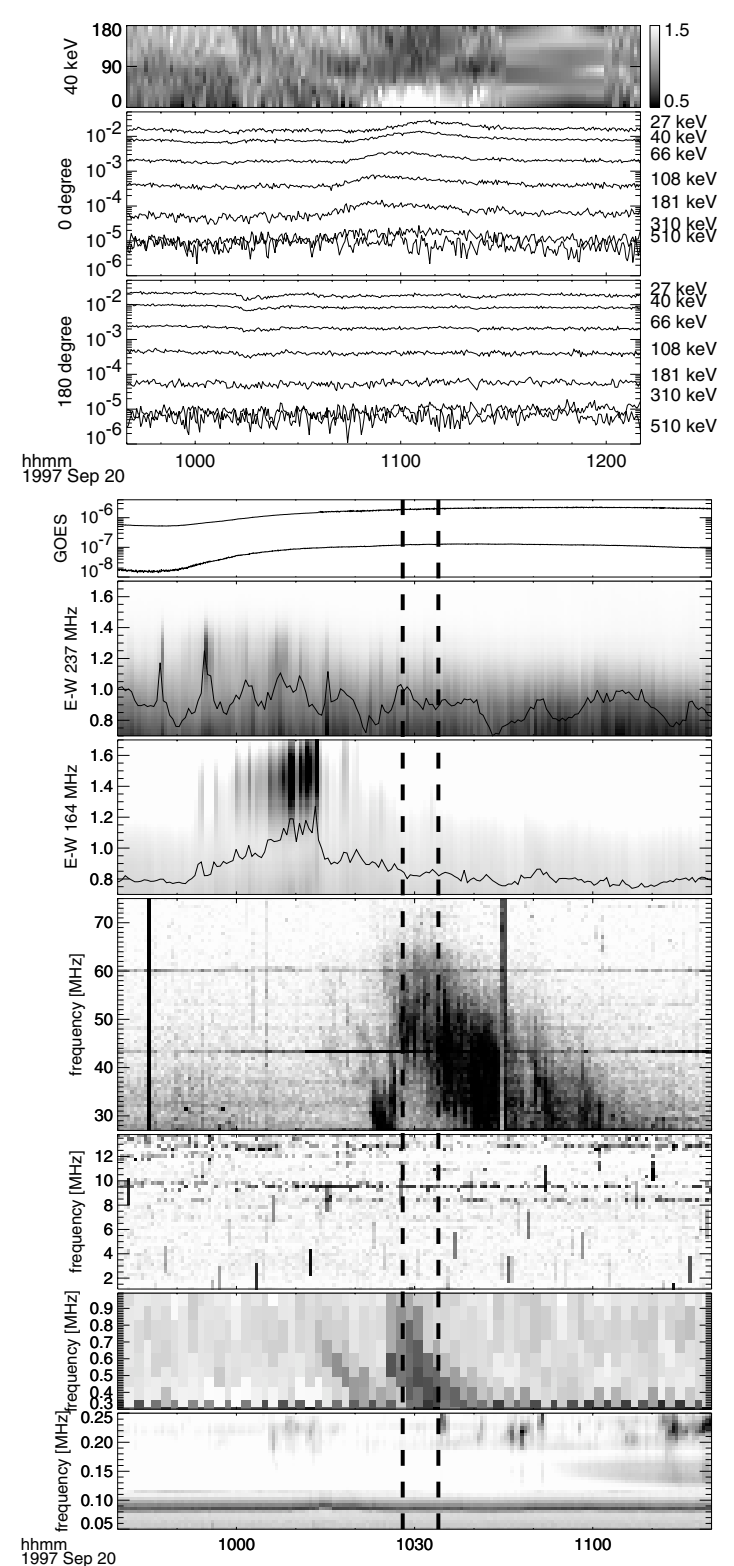

Fig. A.3. 1997 September 20: time history of electrons detected by Wind/3DP (top) and of electromagnetic emissions (see Fig. 1).

motions at fixed frequencies are consistent with plasma emission from expanding magnetic structures or from newly forming structures at increasing altitude, e.g. newly reconnected loops. In both cases electrons radiate in an ambient plasma of decreasing electron density, hence decreasing plasma frequency. The sharp low-frequency cutoff suggests the bulk of the radiating energetic electrons was confined in the coronal structures. Some escaping electrons or electrons accelerated on open field lines may have been at the origin of the weak DH type III bursts seen by WAVES during this phase.

The brightest DH type III burst during this event started with a new metric type IV emission near 13:25 UT. The type IV emission was not the continuation of the previous one, with a new brightness enhancement, but started at a different place as prominently seen in the 1D time histories at $164 \mathrm{MHz}$ (Fig. A.4a). It hence reveals a different episode of electron acceleration in the corona. The metre wave emission was a type IV continuum, and not the high-frequency continuation of the DH type III burst. A typical configuration of metre wave sources in this phase of the event is illustrated in Fig. A.4b, where the iso-intensity contours at half maximum at 432, 327, 237 and $164 \mathrm{MHz}$ are overplotted on a full-Sun image taken by $S X T /$ Yohkoh about $50 \mathrm{~min}$ earlier. The radio sources were at least double at all frequencies $\leqslant 327 \mathrm{MHz}$. The southernmost source overlay a strongly non-radial, southwestward oriented coronal structure in the SXT image, while the northern sources projected above a more radially oriented loop. Jets in $\mathrm{H} \alpha$, the Fe XIV (green) line and soft X-rays were seen in this structure between roughly 13:15 and 13:30 UT, i.e. during the moving radio emission, by Bagalá et al. (2001) who give a detailed discussion of the coronal evolution during the event (cf. also Maia \& Pick 2004). The source centroid positions of the DH type III burst at frequencies above $200 \mathrm{kHz}$, where the burst could be distinguished from subsequent ones, are plotted by plus-signs in Fig. A.4c. The trajectory outlined by this plot (arrow labelled "(1)") extended north of the ecliptic plane. Hence the timing and the location of the type III emission suggest that the electron beams were related to the acceleration of electrons producing the metric type IV emission. Since the type III burst did not extend into the low frequency band of WAVES (bottom panel of Fig. A.4a), the electron beams producing the bright DH type III burst probably travelled along flux tubes which did not intercept the spacecraft.

At the start of the release window of near-relativistic electrons near 13:35 UT a new moving metre wave continuum (moving type IV burst) appeared at 237 and $164 \mathrm{MHz}$ (Fig. A.4a), but not at the higher frequencies. It is clearly distinguished from the previous sources in projection on the solar south-north direction. A snapshot map near its start is plotted in Fig. A.4d. The southern component of the $237 \mathrm{MHz}$ source (light contour) is a residual of previous emission. The moving radio source rose about radially into the corona above the northern soft X-ray loop. Yohkoh/SXT observed the ejection of hot material during this interval (13:32-13:40; McKenzie 2000).

A second bright DH type III burst started near $2 \mathrm{MHz}$ shortly after the release window. The asterisks in Fig. A.4c mark the centroid positions at successively lower frequencies from $940 \mathrm{kHz}$ close to the Sun to $136 \mathrm{kHz}$. The arrow (labelled "(2)") shows the direction from high to low frequencies. The azimuth and elevation were measured at each frequency at the time of the peak of the burst. Although several type III bursts were superposed upon each other during this event, we can suppose that a single burst position dominates at the peak, so that the method provides a reliable indication of the overall direction of propagation. The trajectory extrapolated back to the flaring active region. The radio sources comprise the ecliptic plane, but the global trend of increasing centroid position with decreasing frequency suggests that the type III emitting electrons propagated within flux tubes which eventually bended southward of the ecliptic and away from the spacecraft. This is consistent with the non detection of Langmuir waves at Wind, although the type III emission reached the plasma line (after the interval plotted in the bottom panel of Fig. A.4a). 
K.-L. Klein et al.: Coronal phenomena at the release of solar energetic electron events, Online Material p 6
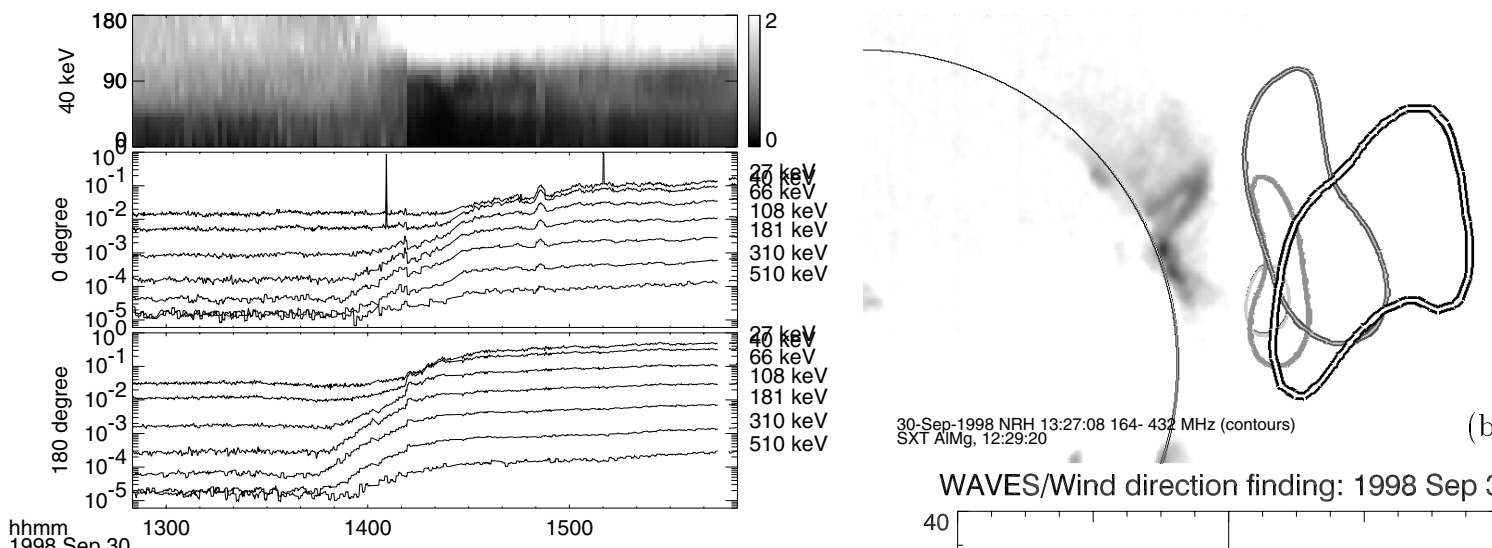

(b)
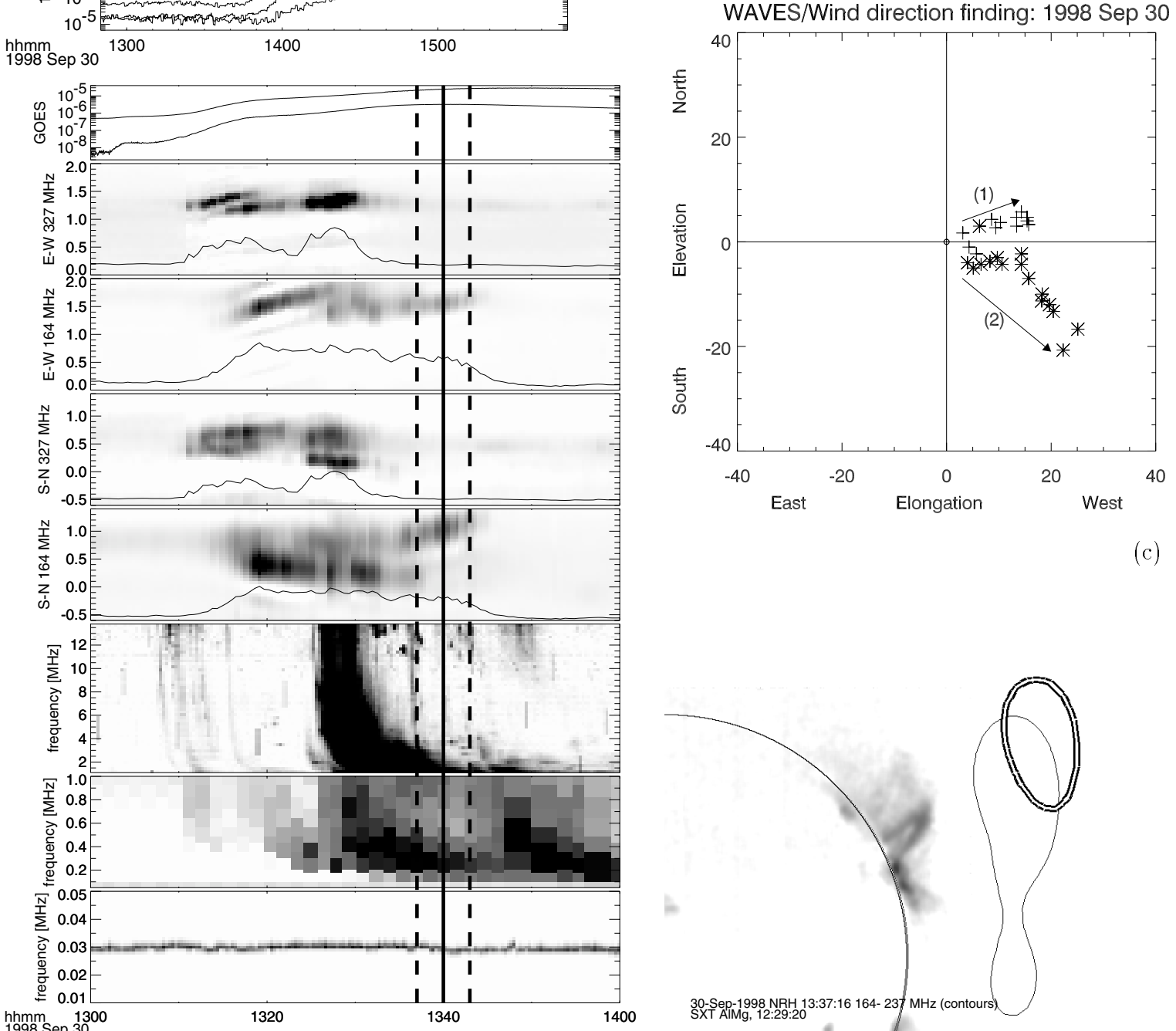

(a)

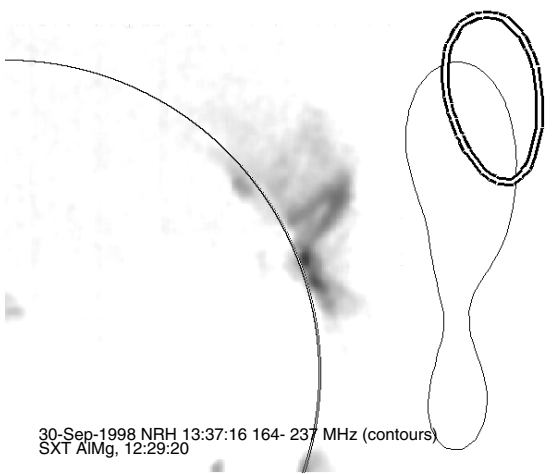

(d)

Fig. A.4. 1998 September 30: a) time history of the metric-to-decametric whole Sun radio flux density, compared with the release window of energetic electrons (vertical lines). (b), d)) Metric radio sources represented by half-maximum contours at the time of the DH type III burst (top) and during the moving type IV bursts (bottom; 237, $164 \mathrm{MHz}$ only). c) the source centroids at frequencies $\leq 940 \mathrm{kHz}$. Arrows show the direction from high to low frequencies. Plus signs refer to the initial type III burst (arrow 1; positions at 940-224 kHz), asterisks to the one starting below $2 \mathrm{MHz}$ just after the electron release window (2; positions $940-136 \mathrm{kHz}$ ). The source radii increase from $\sim 15^{\circ}$ at $940 \mathrm{kHz}$ to $60^{\circ}$ at $136 \mathrm{kHz}$.

In conclusion, there were multiple episodes of electron release to interplanetary space during this event. While the type III emitting beams did not reach Wind, the electrons detected by $3 D P$ were most likely accelerated at the time of the moving type IV burst at metre wavelengths, i.e. during a period of magnetic restructuring of the corona. The different conclu- sion by Maia \& Pick (2004) who identified the electron release seen at ACE with the second type III burst, seems to be contradicted by the interplanetary type III source locations and the non-detection of Langmuir waves. But their timing of the electron release is consistent with ours, to within the statistical uncertainties. 
K.-L. Klein et al.: Coronal phenomena at the release of solar energetic electron events, Online Material $p 7$

Table A.1. 3DP events with no obvious NRH counterpart in the western hemisphere.

\begin{tabular}{|c|c|c|c|c|c|c|}
\hline $\begin{array}{l}\text { Release } \\
\text { window }\end{array}$ & $\begin{array}{r}E \text { range } \\
{[\mathrm{keV}]}\end{array}$ & $\begin{array}{r}(0-14) \mathrm{MHz} \\
\text { (WAVES) }\end{array}$ & $\begin{array}{r}(20-70) \mathrm{MHz} \\
(\mathrm{NDA})\end{array}$ & $\begin{array}{r}\geq 164 \mathrm{MHz} \\
(\mathrm{NRH})\end{array}$ & $\begin{array}{l}\text { Flare position } \\
\text { SXR (GOES) }\end{array}$ & Comment \\
\hline 1997 Apr. 7 & $\sim 1-\geq 500$ & III $>14 \mathrm{MHz}$ & III 20-70 & complex & S29E20 2N 13:53 & flare \& $\mathrm{m}-\lambda$ \\
\hline $14: 21-14: 27$ & & $13: 57-14: 36$ & $13: 56-14: 34$ & $13: 56-15: 15$ & C6.8 13:54 14:03 & in $\mathrm{E}$ hemisphere \\
\hline$(-15+30)$ & & $\begin{array}{l}\text { II 14:30@11 } \\
\text { No L waves }\end{array}$ & & $0.4135^{\circ}$ & & \\
\hline Oct. 7 & $\sim 30-300$ & III $6 \mathrm{MHz}$ & II & II & None reported & occulted flare; \\
\hline $13: 21-13: 33$ & & $12: 50$ & $12: 47-12: 55$ & $12: 47-12: 51$ & No event & ambiguous \\
\hline$(+31+43)$ & & L waves & & $1.6245^{\circ}$ & & association \\
\hline Dec. 18 & $\sim 30-100$ & III $>14 \mathrm{MHz}$ & No data & III/DCIM & None reported & $m-\lambda$ in $E$ \\
\hline $12: 22-12: 28$ & & $12: 04$ & & $12: 19-12: 20$ & B1.2 12:19 12:25 & hemisphere \\
\hline$(+18+24)$ & & No L waves & & $0.265^{\circ}$ & & \\
\hline 1998 May 19 & $\sim 30-300$ & III $>14 \mathrm{MHz}$ & No data & Noise storm & Filament eruption & ongoing noise \\
\hline $\begin{array}{r}10: 04-10: 10 ? \\
(-8+19)\end{array}$ & & $\begin{array}{l}\text { 09:51-10:12 } \\
\text { II 10:01@14 }\end{array}$ & & E hemisph. & $\begin{array}{l}\text { N29 W46 } 0926 \text { U } \\
\text { B7.9 10:10 10:15 }\end{array}$ & $\begin{array}{l}\text { storm, } \\
\text { E hemisph. }\end{array}$ \\
\hline $\begin{array}{r}\text { Jul. 13 } \\
10: 23-10: 27 \\
(+16+20)\end{array}$ & $\sim 30-100$ & $\begin{array}{r}\text { No L waves } \\
\text { III } 5 \mathrm{MHz} \\
\text { 10:02, 10:07 } \\
\text { L waves }\end{array}$ & $\begin{array}{r}\text { II } 20-70 \\
09: 36-09: 51\end{array}$ & quiet & $\begin{array}{l}\text { None reported } \\
\text { B2.7 10:26 10:31 }\end{array}$ & $\begin{array}{l}\text { No radio } \\
\text { counterpart }\end{array}$ \\
\hline $\begin{array}{r}\text { 1999 Jan. } 24 \\
11: 19-11: 29 \\
(-31+20)\end{array}$ & $\sim 30-300$ & $\begin{array}{r}\mathrm{III}>14 \mathrm{MHz} \\
11: 09-11: 50 \\
\text { L waves; BS }\end{array}$ & No data & $\begin{array}{r}\text { Noise storm } \\
\text { enh. } \sim 11: 20 \\
1.4260^{\circ}\end{array}$ & $\begin{array}{l}\text { N21W30 SF 11:06 } \\
\text { C3.9 11:03 11:25 }\end{array}$ & $\begin{array}{l}\text { RW during burst } \\
\text { group within noise } \\
\text { storm }\end{array}$ \\
\hline 2000 Apr. 2 & $\sim 30-100$ & $\begin{array}{r}\text { III }>14 \mathrm{MHz} \\
09 \cdot 49-09 \cdot 56\end{array}$ & No data & Noise st. enh. & $\begin{array}{l}\text { EIT brightenings } \\
09 \cdot 48\end{array}$ & RW near end of \\
\hline $\begin{array}{r}09: 56-10: 02 \\
(0+13)\end{array}$ & & $\begin{array}{r}\text { 09:49-09:56 } \\
\text { L waves }\end{array}$ & & $\begin{array}{r}09: 40-10: 10 \\
0.8290^{\circ}\end{array}$ & $\begin{array}{l}09: 48 \\
\text { C1.7 09:41 09:49 }\end{array}$ & $\begin{array}{l}\text { noise storm } \\
\text { enhancement }\end{array}$ \\
\hline $\begin{array}{r}\text { Apr. } 27 \\
14: 18-14: 38\end{array}$ & $\sim 1-100$ & $\begin{array}{r}\text { III storm } \\
\sim 12 \mathrm{MHz} \\
\text { II/IV 14:40@14 } \\
\text { No L waves }\end{array}$ & No data & $\begin{array}{r}\text { Noise storm } \\
\sim 14 \mathrm{UT} \\
0.50^{\circ}\end{array}$ & $\begin{array}{l}\text { Promin. eruption } \\
\text { N17 W90 14:25E } \\
\text { B9.8 14:04 14:40 }\end{array}$ & $\begin{array}{l}\text { Noise storm } \\
\text { near CM: } \\
\text { Doubtful assoc. }\end{array}$ \\
\hline Oct. 27 & $\sim 30-100$ & III $>14 \mathrm{MHz}$ & III $20-70$ & Burst & EIT brightening & RW with late \\
\hline $11: 39-11: 47$ & & $11: 32,11: 46$ & $\sim 11: 30-12: 30$ & $11: 19$ & NW limb 11:28 & III $<5 \mathrm{MHz}$ \\
\hline$(-7+15)$ & & $\begin{array}{r}\text { II 12:30@14 } \\
\text { No L waves }\end{array}$ & & $1.7295^{\circ}$ & $\begin{array}{l}\text { C8.2 11:14 11:27 } \\
\text { C9.7 11:55 12:03 }\end{array}$ & \\
\hline $\begin{array}{r}2001 \text { May } 7 \\
09: 46-09: 52 \\
(-4+31)\end{array}$ & $\sim 1-300$ & $\begin{array}{r}\text { III } 6 \mathrm{MHz} \\
09: 21-09: 50 \\
\text { L waves }\end{array}$ & No event & $\begin{array}{r}\text { Noise storm } \\
09: 30-10: 10 \\
0.8315^{\circ}\end{array}$ & $\begin{array}{l}\text { None reported } \\
\text { No event }\end{array}$ & $\begin{array}{l}\text { RW during burst } \\
\text { group within } \\
\text { noise storm }\end{array}$ \\
\hline
\end{tabular}

While the moving coronal radio sources were in progress, a type II burst was distinguished at and below $60 \mathrm{MHz}$ (Reiner et al. 2000). It started near 13:22:30 UT, more than ten minutes before the bright DH type III bursts, but well after the initial metric continuum, and proceeded into the high frequency band of WAVES during the bright DH type III burst. The timing with respect to the continuum and the fact that the type II burst followed the low-frequency border of the continuum suggest that the type II emission came from a shock driven by the expanding coronal structures revealed by the metric type IV burst sources. Such a relationship between metric type II emission and expanding active region structures was shown earlier for simpler events (Klein et al. 1999; Klassen et al. 2003). The bright DH type III bursts can be ascribed to the type II shock, from which they may have emanated, but, as discussed above, they also occurred at the time of bright new metric radio sources far lower in the corona, and the onset of associated emission extending well above $1 \mathrm{GHz}$ (Reiner et al. 2000). Such an ambiguous association with other radio features is usual during the so-called "shock-associated" type III events, but in this case the observations suggest a secondary role of the shock. The re- lease of the near-relativistic electrons detected at Wind started at some time during this type II burst, but without any more specific temporal relationship.

\section{A.3. Electron events with no NRH counterpart}

The electron events with no associated metre wave emission at or above $164 \mathrm{MHz}$ are listed in Table A.1. The format is similar to Tables 1 and 2, but no CME information is given. The last column of this Table gives a short comment on the relationship between the electron release and the radio emission. There are several reasons why we did not associate these electron releases with metric radio emission:

- Two events had no radio counterpart at any frequency near the release window: 1997 Oct. 7, 1998 Jul. 13. The first event is probably associated with a flare well behind the western limb, so that relevant emissions were likely occulted (cf. Klein et al. 2003b).

- In two events radio emission was observed at lower frequencies than $164 \mathrm{MHz}$ during the release window: 1998 
May 19, 2000 Oct. 27. The former event was associated with a DH type II burst (cf. Klassen et al. 2002). During the release window of the second event a DH type III burst started near $5 \mathrm{MHz}$, with no signature of a type II shock at that time.

- In three events (1999 Jan. 24, 2000 Apr. 2, 2001 May 7) long lasting metric emission (noise storm) existed in the western hemisphere, but showed no peculiar feature during the release window. It cannot be excluded that part of this emission was accompanied by electron release into interplanetary space, though.

- In three events a specific radio feature did exist near the time of the electron release, and would have been associated with it, were it not for the fact that the radio source was located in the eastern hemisphere: 1997 Apr. 7 (cf. Krucker et al. 1999), 1997 Dec. 18, 2000 Apr. 27. 